\title{
FIVE EMBEDDINGS OF ONE SIMPLE GROUP
}

\author{
IVAN CHELTSOV AND CONSTANTIN SHRAMOV
}

\begin{abstract}
We propose a new method to study birational maps between Fano varieties based on multiplier ideal sheaves. Using this method, we prove equivariant birational rigidity of four Fano threefolds acted on by the group $A_{6}$. As an application, we obtain that $\operatorname{Bir}\left(\mathbb{P}^{3}\right)$ has at least five non-conjugate subgroups isomorphic to $\mathrm{A}_{6}$.
\end{abstract}

\section{CONTEnts}

1. Introduction

2. Preliminaries

3. Alternating group

4. Projective space $\quad 15$

5. Segre cubic $\quad 23$

6. Quadric threefold $\quad 27$

Appendix A. Klein cubic threefold $\quad 30$

Appendix B. Del Pezzo fibrations $\quad 32$

References

We assume that all varieties are projective, normal, and defined over $\mathbb{C}$.

\section{INTRODUCTION}

The group of birational automorphisms of the projective plane $\mathbb{P}^{2}$, also known as the Cremona group $\mathrm{Cr}_{2}(\mathbb{C})$, is a classical object in algebraic geometry. Classifying finite subgroups in $\mathrm{Cr}_{2}(\mathbb{C})$ up to conjugacy is an important research direction originating in the works of Kantor, Bertini, Wiman and others. This classification has been almost completed in [16]. In particular, we know how to decide whether two finite isomorphic subgroups in $\mathrm{Cr}_{2}(\mathbb{C})$ are conjugate or not.

The group of birational automorphisms of the projective space $\mathbb{P}^{3}$, the Cremona group $\mathrm{Cr}_{3}(\mathbb{C})$, is poorly understood. We know so little about finite subgroups in $\mathrm{Cr}_{3}(\mathbb{C})$ that Serre asked

Question 1.1 ([40, Question 6.0]). Does there exist a finite group which is not embeddable in $\mathrm{Cr}_{3}(\mathbb{C})$ ?

It is well-know that most of finite groups are not embeddable in $\mathrm{Cr}_{2}(\mathbb{C})$. For example, the only finite simple non-abelian subgroup in $\mathrm{Cr}_{2}(\mathbb{C})$ are $\mathrm{A}_{5}, \mathrm{~A}_{6}, \mathrm{PSL}_{2}\left(\mathbb{F}_{7}\right)$ (see [16]). Prokhorov explicitly answered Question 1.1 by proving the following

Theorem 1.2 ([38, Theorem 1.3]). Let $\bar{G}$ be a finite simple non-abelian subgroup in $\mathrm{Cr}_{3}(\mathbb{C})$. Then $\bar{G}$ is one of the following groups: $\mathrm{A}_{5}, \mathrm{~A}_{6}, \mathrm{PSL}_{2}\left(\mathbb{F}_{7}\right), \mathrm{A}_{7}, \mathrm{SL}_{2}\left(\mathbb{F}_{8}\right), \mathrm{PSp}_{4}\left(\mathbb{F}_{3}\right)$. All the possibilities occur.

During a workshop "Subgroups of Cremona groups" at Edinburgh in Spring 2010, Serre posed

The first author was supported by the grants NSF DMS-0701465 and EPSRC EP/E048412/1, the second author was supported by the grants RFFI 08-01-00395-a, NSh-1987.2008.1 and EPSRC EP/E048412/1. 
Question 1.3. What are normalizers in $\mathrm{Cr}_{3}(\mathbb{C})$ of finite simple non-abelian subgroup in $\mathrm{Cr}_{3}(\mathbb{C})$ ?

An answer to Question 1.3 depends on classification of finite non-abelian simple subgroups in $\mathrm{Cr}_{3}(\mathbb{C})$ up to conjugation. But Theorem 1.2 gives such classification only up to isomorphism. So before trying to answer Question 1.3 , we must first consider

Question 1.4. How to decide whether two finite isomorphic subgroups in $\mathrm{Cr}_{3}(\mathbb{C})$ are conjugate or not?

With a very few exceptions (see [31, 8], 38]), we do not know how to answer Question 1.4 in full generality. In this paper, we show a new technique that can be used to answer both Questions 1.3 and 1.4 in some cases. In particular, we use this technique to prove

Theorem 1.5. Up to conjugation, there are at least 5 subgroups in $\mathrm{Cr}_{3}(\mathbb{C})$ that are isomorphic to $\mathrm{A}_{6}$. For three of these non-conjugated subgroups, the normalizer in $\mathrm{Cr}_{3}(\mathbb{C})$ is $\mathrm{S}_{6}$, and for one is the free product of $S_{6}$ and $S_{6}$ with an amalgamated subgroup $A_{6}$.

Let us show how to translate Questions 1.4 and 1.3 into a geometric language. Let $\bar{G}$ be a finite subgroup, and let $\tau: \bar{G} \rightarrow \mathrm{Cr}_{3}(\mathbb{C})$ be a monomorphism. It is well-known (see [38, Section 4.2]) that there is a birational map $\xi: V \rightarrow \mathbb{P}^{3}$ such that

- the threefold $V$ is normal and has terminal singularities,

- there exists a monomorphism $v: \bar{G} \rightarrow \operatorname{Aut}(V)$,

- for every element $g \in \bar{G}$, we have $\tau(g)=\xi \circ v(g) \circ \xi^{-1}$,

- there exists a $v(\bar{G})$-Mori fibration $\pi: V \rightarrow S$, i. e. a non-birational $v(\bar{G})$-equivariant surjective morphism with connected fibers such that the divisor $-K_{V}$ is $\pi$-ample and for every $v(\bar{G})$-invariant Weil divisor $D$ on $V$, there is $\delta \in \mathbb{Q}$ such that

$$
\delta K_{V}+D \sim_{\mathbb{Q}} \pi^{*}(H)
$$

for some $\mathbb{Q}$-Cartier divisor $H$ on the variety $S$.

Definition 1.6. The quadruple $(V, \xi, v, \pi)$ is a Mori regularization of the pair $(\bar{G}, \tau)$.

Theorem 1.7. The normalizer of the group $\tau(\bar{G})$ in $\mathrm{Cr}_{3}(\mathbb{C})$ is isomorphic to the group of all $v(\bar{G})$-equivariant birational automorphisms of the variety $V$.

Proof. The proof is obvious and left to the reader.

Let $\tau^{\prime}: \bar{G} \rightarrow \mathrm{Cr}_{3}(\mathbb{C})$ be another monomorphism, and let $\left(V^{\prime}, \xi^{\prime}, v^{\prime}, \pi^{\prime}\right)$ be a Mori regularization of the pair $\left(\bar{G}, \tau^{\prime}\right)$.

Theorem 1.8 ([16, Lemma 3.4]). The following assertions are equivalent:

- the subgroups $\tau(\bar{G})$ and $\tau^{\prime}(\bar{G})$ are conjugate in $\mathrm{Cr}_{3}(\mathbb{C})$,

- there is a birational map $\rho: V \rightarrow V^{\prime}$ such that for every $g \in \bar{G}$ there is $g^{\prime} \in \bar{G}$ such that

$$
v^{\prime}\left(g^{\prime}\right)=\rho \circ v(g) \circ \rho^{-1} \in \operatorname{Aut}\left(V^{\prime}\right) .
$$

Thus, the subgroups $\tau(\bar{G})$ and $\tau^{\prime}(\bar{G})$ are conjugate in $\mathrm{Cr}_{3}(\mathbb{C})$ if and only if there exists a $\bar{G}$-equivariant birational map $V \rightarrow V^{\prime}$ with respect to the actions of the group $\bar{G}$ on $V$ and $V^{\prime}$ induced by the monomorphisms $v$ and $v^{\prime}$. However, to prove or disprove the existence of such birational map is not easy in general. The following two definitions help to deal with this in the case when $\pi(V)=S$ is a point, i.e. in the case when $V$ is a normal Fano variety with terminal singularities such that the $v(\bar{G})$-invariant subgroup in $\mathrm{Cl}(V) \otimes \mathbb{Q}$ is generated by $-K_{V}$.

Definition 1.9 (cf. [7, Definition 0.3.3]). If $S$ is a point, then $V$ is called $v(\bar{G})$-birationally rigid if for every Mori regularization $\left(V^{\prime}, \xi^{\prime}, v^{\prime}, \pi^{\prime}\right)$ of the pair $(\bar{G}, \tau)$, we have $V^{\prime} \cong V, \pi^{\prime}\left(V^{\prime}\right)$ is a point, and the subgroups $v(\bar{G})$ and $v^{\prime}(\bar{G})$ are conjugate in $\operatorname{Aut}(V) \cong \operatorname{Aut}\left(V^{\prime}\right)$. 
Definition 1.10 (cf. [7, Definition 0.3.4]). If $S$ is a point, then $V$ is called $v(\bar{G})$-birationally superrigid if for every Mori regularization $\left(V^{\prime}, \xi^{\prime}, v^{\prime}, \pi^{\prime}\right)$ of the pair $(\bar{G}, \tau)$, the map $\xi^{-1} \circ \xi^{\prime}$ is biregular.

From now on we will identify the group $\bar{G}$ with its image $v(\bar{G})$ to simplify the notation. Note that if $S$ is a point and $V$ is $\bar{G}$-birationally superrigid, then it follows from Definitions 1.9 and 1.10 that $V$ is $\bar{G}$-birationally rigid. Denote by $\operatorname{Aut}^{\bar{G}}(V)$ (by $\operatorname{Bir}^{\bar{G}}(V)$, respectively) the groups of $\bar{G}$-equivariant biregular (birational, respectively) self-maps of $V$. Note also that the group $\operatorname{Aut}^{\bar{G}}(V)$ (the group $\operatorname{Bir}^{\bar{G}}(V)$, respectively) coincides with the normalizer of $\bar{G}$ in $\operatorname{Aut}(V)$ (in $\operatorname{Bir}(V)$, respectively).

Corollary 1.11. Suppose that $S$ is a point, the threefold $V$ is $\bar{G}$-birationally rigid, and $V \neq V^{\prime}$. Then $\tau(\bar{G})$ and $\tau^{\prime}(\bar{G})$ are not conjugate in $\mathrm{Cr}_{3}(\mathbb{C})$.

Corollary 1.12. Suppose that $S$ is a point, and $V$ is $\bar{G}$-birationally superrigid. Then the normalizer of the group $\tau(\bar{G})$ in $\mathrm{Cr}_{3}(\mathbb{C})$ is isomorphic to $\operatorname{Aut}^{\bar{G}}(V)$.

Let us consider examples of Mori regularizations in the case when $\bar{G}$ is one of the following groups: $\mathrm{A}_{7}, \mathrm{SL}_{2}\left(\mathbb{F}_{8}\right)$, and $\mathrm{PSp}_{4}\left(\mathbb{F}_{3}\right)$. Note that in the latter case, the variety $S$ must be a point, since $\mathrm{A}_{7}, \mathrm{SL}_{2}\left(\mathbb{F}_{8}\right)$, and $\mathrm{PSp}_{4}\left(\mathbb{F}_{3}\right)$ are not embeddable in $\mathrm{Cr}_{2}(\mathbb{C})$.

Example 1.13. If $\bar{G}=\mathrm{A}_{7}$ or $\bar{G} \cong \operatorname{PSp}_{4}\left(\mathbb{F}_{3}\right)$, then there is a monomorphism $v: \bar{G} \rightarrow \operatorname{Aut}\left(\mathbb{P}^{3}\right)$ (see [15]).

Example 1.14 ([38, Example 2.11]). Put $\bar{G}=\mathrm{SL}_{2}\left(\mathbb{F}_{8}\right)$. One can show that there is a monomorphism $\alpha: \bar{G} \rightarrow \operatorname{Aut}(\operatorname{LGr}(4,9))$, and $\operatorname{Pic}(\operatorname{LGr}(4,9))=\mathbb{Z}[H]$, where $H$ is an ample divisor such that $|H|$ gives an embedding $\zeta: \operatorname{LGr}(4,9) \rightarrow \mathbb{P}^{15}$, which implies that the monomorphism $\alpha$ induces a monomorphism $\beta: \bar{G} \rightarrow \operatorname{Aut}\left(\mathbb{P}^{15}\right)$. Put $V=\zeta(\operatorname{LGr}(4,9)) \cap \Pi$, where $\Pi$ is the unique $\beta(\bar{G})$-invariant linear subspace $\Pi \subset \mathbb{P}^{15}$ such that $\operatorname{dim}(\Pi)=8$. Then $V$ is a smooth Fano threefold such that $\operatorname{Pic}(V) \cong \mathbb{Z}$ and $-K_{V}^{3}=12$ (see [35]), and $V$ is rational (see [25, Corollary 4.4.12]). The monomorphism $\beta$ induces an isomorphism $v: \bar{G} \rightarrow \operatorname{Aut}(V)$.

Example 1.15. Suppose that $V$ is a complete intersection in $\mathbb{P}^{5}$ that is given by

$$
\sum_{i=0}^{5} x_{i}=\sigma_{4}\left(x_{0}, x_{1}, x_{2}, x_{3}, x_{4}, x_{5}\right)=0 \subset \mathbb{P}^{5} \cong \operatorname{Proj}\left(\mathbb{C}\left[x_{0}, x_{1}, x_{2}, x_{3}, x_{4}, x_{5}\right]\right),
$$

where $\sigma_{4}$ is the elementary symmetric form of degree 4 . Put $\bar{G}=\operatorname{PSp}_{4}\left(\mathbb{F}_{3}\right)$. Then there is a monomorphism $v: \bar{G} \rightarrow \operatorname{Aut}(V)$ (see [23, Chapter 5]). It is known that the threefold $V$ is rational (see [23, Section 5.2.7]), and the $\bar{G}$-invariant subgroup in the group $\mathrm{Cl}(V)$ is $\mathbb{Z}$ (cf. Theorem 1.20).

The threefold constructed in Example 1.15 is known as the Burkhardt quartic (see [23]).

Theorem 1.16 ([38, Theorem 1.5], 4]). Suppose that $\bar{G}$ is one of the following subgroup: $A_{7}$, $\mathrm{SL}_{2}\left(\mathbb{F}_{8}\right)$, or $\mathrm{PSp}_{4}\left(\mathbb{F}_{3}\right)$. Let $V$ be a normal rational threefold with at most terminal singularities that admits a faithful action of the group $\bar{G}$ such that there exists a $\bar{G}$-Mori fibration $\pi: V \rightarrow S$. Then $S$ is a point, and

- if $\bar{G} \cong \mathrm{A}_{7}$, then $V \cong \mathbb{P}^{3}$ (see Example 1.13),

- if $\bar{G} \cong \mathrm{SL}_{2}\left(\mathbb{F}_{8}\right)$, then $V$ is the threefold constructed in Example 1.14.

- if $\bar{G} \cong \operatorname{PSp}_{4}\left(\mathbb{F}_{3}\right)$, then either $V \cong \mathbb{P}^{3}$ or $V$ is the threefold constructed in Example 1.15.

Now let us consider examples of Mori regularizations in the case when $\bar{G} \cong \mathrm{A}_{6}$.

Example 1.17. Put $V=\mathbb{P}^{3}$ and $\bar{G}=\mathrm{A}_{6}$. Then there exists a monomorphism $2 . \mathrm{A}_{6} \rightarrow \mathrm{SL}_{4}(\mathbb{C})$ (see [15]), which induces a monomorphism $v: \bar{G} \rightarrow \operatorname{Aut}(V) \cong \mathrm{PGL}_{4}(\mathbb{C})$. Furthermore, up to conjugation this is the unique subgroup isomorphic to $\mathrm{A}_{6}$ in $\mathrm{PGL}_{4}(\mathbb{C})$. 
Example 1.18. Put $\bar{G}=\mathrm{A}_{6}$. Then there exists a monomorphism $\bar{G} \rightarrow \mathrm{SO}_{5}(\mathbb{R})$, which implies the existence of a monomorphism $v: \bar{G} \rightarrow \operatorname{Aut}(V)$, where $V$ is a smooth quadric threefold.

Example 1.19. Put $\bar{G}=\mathrm{A}_{6}$. Suppose that $V$ is the complete intersection

$$
\sum_{i=0}^{5} x_{i}=\sum_{i=0}^{5} x_{i}^{3}=0 \subset \mathbb{P}^{5} \cong \operatorname{Proj}\left(\mathbb{C}\left[x_{0}, x_{1}, x_{2}, x_{3}, x_{4}, x_{5}\right]\right) \text {. }
$$

Then $V$ has exactly 10 isolated ordinary double points (and hence $V$ is rational), and there exists a natural monomorphism $v: \bar{G} \rightarrow \operatorname{Aut}(V)$. Furthermore, one has $\mathrm{Cl}(V) \cong \mathbb{Z}^{6}$ (see [18], [19]), which implies that the $\bar{G}$-invariant subgroup of the group $\mathrm{Cl}(V)$ is $\mathbb{Z}$.

The threefold constructed in Example 1.19] is known as the Segre cubic (see [23, Section 3.2]). Let us show how to prove that $V$ is $\bar{G}$-birational superrigid in the case when $S$ is a point.

Theorem 1.20. Put $\bar{G}=\mathrm{A}_{6}$. Suppose that $V$ is the threefold constructed in Example 1.15. Then $\operatorname{rkCl}^{\bar{G}}(V)=1$, the variety $V$ is $\bar{G}$-birationally superrigid, and $\operatorname{Bir}^{\bar{G}}(V) \cong \mathrm{S}_{6}$.

Proof. The required assertions are probably well-known to experts. But we failed to find any relevant reference. The fact that the $\bar{G}$-invariant subgroup of the group $\mathrm{Cl}(V)$ is $\mathbb{Z}$ follows from the isomorphism $V / \bar{F} \cong \mathbb{P}(1,2,2,3)$, where $\bar{F}$ is a subgroup in $\bar{G}$ such that $\bar{F} \cong \mathrm{S}_{4}$ and $\bar{F}$ fixes a point in $\operatorname{Sing}(V)$. The isomorphism $\operatorname{Bir}^{\bar{G}}(V) \cong \mathrm{S}_{6}$ follows from $\bar{G}$-birational superrigidity of the threeefold $V$, Corollary 1.12, and classification of primitive subgroups in $\mathrm{SL}_{5}(\mathbb{C})$ (see [17]). The fact that $V$ is $\bar{G}$-birationally superrigid easily follows from the proof of [33, Theorem 5] (cf. [42, Section 9]), but we decided to prove this here to illustrate how to proof $\bar{G}$-birational superrigidity using only classical technique that goes back to [24].

Suppose that $V$ is not $\bar{G}$-birationally superrigid. Then [12, Theorem 4.2] implies the existence of a $\bar{G}$-invariant linear system $\mathcal{M}$ on the threefold $V$ such that $\mathcal{M}$ does not have fixed components, and the log pair $(V, \lambda \mathcal{M})$ is not canonical, where $\lambda \in \mathbb{Q}$ is such that $K_{V}+\lambda \mathcal{M} \sim_{\mathbb{Q}} 0$.

Let $M_{1}$ and $M_{2}$ be sufficiently general surfaces in the linear system $\mathcal{M}$, and let $H$ be a general surface in $\left|-K_{V}\right|$. Consider $V$ as a quartic threefold in $\mathbb{P}^{4}$.

Suppose that there is an irreducible curve $C \subset V$ such that the $\log$ pair $(V, \lambda \mathcal{M})$ is not canonical along the curve $C$. Then the multiplicity of the linear system $|\mathcal{M}|$ along the curve $C$ is greater than $1 / \lambda$ (see e.g. [14, Exercise 6.18]). Let $Z$ be the $\bar{G}$-orbit of the curve $C$. Put $d=-K_{V} \cdot Z$. Then

$$
4 / \lambda^{2}=M_{1} \cdot M_{2} \cdot H \geqslant \operatorname{dmult}_{C}\left(M_{1}\right) \operatorname{mult}_{C}\left(M_{2}\right)>d / \lambda^{2},
$$

which implies that $d \leqslant 3$. So, the curve $Z$ is contained in a hyperplane in $\mathbb{P}^{4}$, which is impossible, because the corresponding five-dimensional representation of the group $\bar{G}$ is irreducible.

Thus, the centers of canonical singularities (see [7, Definition 1.3.8]) of the log pair $(V, \lambda \mathcal{M})$ are points. Take any point $P \in V$ such that the singularities of the log pair $(V, \lambda \mathcal{M})$ are not canonical at the point $P$. Suppose that $P \in V$ is a smooth point, and let $H_{P}$ be a general hyperplane section of $V \subset \mathbb{P}^{4}$ passing through $P$. Then

$$
4 / \lambda^{2}=M_{1} \cdot M_{2} \cdot H_{P} \geqslant \operatorname{mult}_{P}\left(M_{1} \cdot M_{2}\right)>4 / \lambda^{2}
$$

by [13, Corollary 3.4]. The obtained contradiction shows that the quartic $V$ is singular at $P$.

Let $\Sigma$ be the $\bar{G}$-orbit of the point $P \in V$. Then there is a subset $\Gamma \subset \Sigma$ such that $|\Gamma|=4$, and the set $\Gamma$ is not contained in any two-dimensional linear subspace of $\mathbb{P}^{4}$.

Let $\mathcal{Q} \subset\left|-2 K_{V}\right|$ be a linear subsystem that consists of all surfaces in $\left|-2 K_{V}\right|$ that pass through every point of the set $\Gamma$. Then the base locus of the linear system $\mathcal{Q}$ is the set $\Gamma$.

Let $\pi: U \rightarrow V$ be a blow up of the set $\Gamma$, let $\overline{\mathcal{M}}$ be the proper transforms of the linear system $\mathcal{M}$ on the variety $U$, and let $E_{1}, \ldots, E_{4}$ be exceptional divisors of $\pi$. Then there is $m \in \mathbb{Z}$ such that $\overline{\mathcal{M}} \sim \pi^{*}(\mathcal{M})-m \sum_{i=1}^{4} E_{i}$. Moreover, it follows from [7, Theorem 1.7.20] that 
$m>1 / \lambda$ (cf. [13, Theorem 3.10]). Let $\Upsilon$ be the intersection of two sufficiently general surfaces in $\mathcal{Q}$, and let $\bar{\Upsilon}$ be the proper transform of the curve $\Upsilon$ on the threefold $U$. Then

$$
0>8 / \lambda-8 m>8 / \lambda-m \sum_{O \in \Gamma} \operatorname{mult}_{O}(\Upsilon)=\bar{M} \cdot \bar{\Upsilon} \geqslant 0
$$

where $\bar{M}$ is a general surface in $\overline{\mathcal{M}}$. The obtained contradiction completes the proof.

Corollary 1.21. Up to conjugation, there are at least 2 subgroups in $\mathrm{Cr}_{3}(\mathbb{C})$ isomorphic to $\mathrm{A}_{6}$.

Corollary 1.22. Suppose that $\bar{G}=\operatorname{PSp}_{4}\left(\mathbb{F}_{3}\right)$ and $V$ is the threefold constructed in Example 1.15. Then the $\bar{G}$-invariant subgroup of the group $\mathrm{Cl}(V)$ is $\mathbb{Z}$, and $V$ is $\bar{G}$-birationally superrigid.

Now applying Theorem 1.8, Corollary 1.11, and Theorem 1.16, we obtain

Corollary 1.23. Up to conjugation, the group $\mathrm{Cr}_{3}(\mathbb{C})$ contains exactly 1 subgroup that is isomorphic to $\mathrm{SL}_{2}\left(\mathbb{F}_{8}\right)$, exactly 2 subgroups that are isomorphic to $\mathrm{PSp}_{4}\left(\mathbb{F}_{3}\right)$, exactly 1 subgroup that is isomorphic to $\mathrm{A}_{7}$.

The proof of Theorem 1.20 goes back to the classical result of Iskovskikh and Manin about the non-rationality of every smoooth quartic threefold (see [24]). Of course, the quartic threefold constructed in Example 1.15 is not smooth, but this problem is dealt using technique introduced in [13] and [33. So the proof of Theorem 1.20] is a nice illustration how to answer Question 1.4 in one particular case. Unfortunately, this approach is hard to apply to Fano varieties of large anticanonical degree. The main purpose of this paper is to introduce a new technique to prove $\bar{G}$-birational rigidity or $\bar{G}$-birational superrigidity of the threefold $V$ in the case when $S$ is a point. This technique does not always work. But sometimes it does. We will use this technique to prove

Theorem 1.24. Suppose that $\bar{G}=\mathrm{A}_{6}$. If $V \cong \mathbb{P}^{3}$ (cf. Example 1.17), then the threefold $V$ is $\bar{G}$-birationally rigid (but not $\bar{G}$-birationally superrigid), and $\operatorname{Bir}^{\bar{G}}(V)$ is a free product of two copies of $\mathrm{S}_{6}$ with amalgamated subgroup $\mathrm{A}_{6}$. If $V$ is either the Segre cubic or a smooth quadric threefold, then $V$ is $\bar{G}$-birationally superrigid and $\operatorname{Bir}^{\bar{G}}(V) \cong \mathrm{S}_{6}$.

Corollary 1.25. Up to conjugation, there are at least 5 subgroups in $\mathrm{Cr}_{3}(\mathbb{C})$ isomorphic to $\mathrm{A}_{6}$.

Unfortunately, we are unable to classify all subgroups in $\mathrm{Cr}_{3}(\mathbb{C})$ that are isomorphic to $\mathrm{A}_{6}$ up to conjugation similar to what is done in Corollary 1.23 for $\mathrm{A}_{7}, \mathrm{SL}_{2}\left(\mathbb{F}_{8}\right), \mathrm{PSp}_{4}\left(\mathbb{F}_{3}\right)$. The main problem is that $S$ is not necessarily a point if $\bar{G}=\mathrm{A}_{6}$, since $\mathrm{A}_{6}$ is embeddable in $\mathrm{Cr}_{2}(\mathbb{C})$. If $\bar{G}=\mathrm{A}_{6}$ and $\operatorname{dim}(S)=1$, i.e. $\pi: V \rightarrow S$ is a del Pezzo fibration, then $V \cong \mathbb{P}^{2} \times \mathbb{P}^{1}$ by Theorem B.1. However, If $\bar{G}=\mathrm{A}_{6}$ and $\operatorname{dim}(S)=2$, i.e. $\pi: V \rightarrow S$ is a conic bundle, then we do not have any decent description of the threefold $V$ and this is the main reason why we are unable to classify all subgroups in $\mathrm{Cr}_{3}(\mathbb{C})$ that are isomorphic to $\mathrm{A}_{6}$ up to conjugation.

Remark 1.26. Suppose that $\bar{G}=\mathrm{A}_{6}$ and $\operatorname{dim}(S)=2$. Then the monomorphism $v: \bar{G} \rightarrow \operatorname{Aut}(V)$ induces a monomorphism $\iota: \bar{G} \rightarrow \operatorname{Aut}(S)$, because $\pi$ is $\bar{G}$-equivariant and $\bar{G}$ is simple. On the other hand, the group $\bar{G}$ faithfully acts on $\mathbb{P}^{2} \times \mathbb{P}^{1}$ so that its action of the first factor is induced by a three-dimensional representation of the group $3 . \mathrm{A}_{6}$, and its action on the second factor is trivial. Keeping in mind that $V$ is rational, it is very tempting to expect that there always exists a commutative diagram

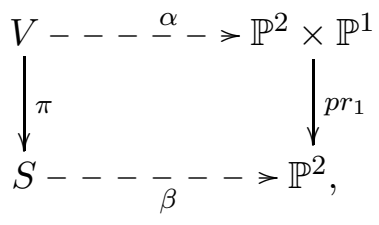


where $\alpha$ is some $\bar{G}$-equivariant birational map, $\beta$ is some $\iota(\bar{G})$-equivariant birational map, and $p r_{1}: \mathbb{P}^{2} \times \mathbb{P}^{1} \rightarrow \mathbb{P}^{2}$ is the projection to the first factor. We do not have any strong evidence for such expectation. But we failed to construct a counter-example either.

Let us describe the strategy how to prove Theorem 1.24, Let $X$ be a Fano threefold with Gorenstein terminal singularities, and let $\bar{G}$ be a subgroup in $\operatorname{Aut}(X)$ such that $\operatorname{rkCl}^{\bar{G}}(X)=1$. We suppose that $X$ is not $\bar{G}$-birationally superrigid and seek for a contradiction. It is well-known that in this case there are a movable $\bar{G}$-invariant linear system $\mathcal{M}$ without fixed components and $\lambda \in \mathbb{Q}$ such that $K_{X}+\lambda \mathcal{M} \sim_{\mathbb{Q}} 0$ and the log pair $(X, \lambda \mathcal{M})$ has non-canonical singularities (see [12, Theorem 4.2], [7, Theorem 1.4.1]). Since $X$ is Gorenstein, the singularities of the log pair $(X, 2 \lambda \mathcal{M})$ must be worse than log canonical by Corollary 2.3. Now, although the last condition is much weaker than non-canonicity of the log pair $(X, \lambda \mathcal{M})$, we are in a position to use the machinery of multiplier ideal sheaves to obtain a contradiction. Namely, we choose $\mu<2 \lambda$ such that the log pair $(X, \mu \mathcal{M})$ is strictly log canonical, and pick up a minimal center $S$ of $\log$ canonical singularities of $(X, \mu \mathcal{M})$ (see Section 2 for definitions). The minimality of the center $S$ implies that the $\bar{G}$-orbit of $S$ is either a finite set, or a disjoint union of irreducible curves. We use Lemma 2.13 to observe that one may assume that every center of log canonical singularities of the log pair $(X, \mu \mathcal{M})$ is $g(S)$ for some $g \in \bar{G}$. Then applying the Nadel-Shokurov vanishing theorem (see Theorem 2.4, [30, Theorem 9.4.8]) we obtain an upper bound on the number of irreducible components of the $\bar{G}$-orbit of $S$. If $S$ is a point, the latter appears to be mostly enough to obtain a contradiction with the structure of $\bar{G}$-orbits on $X$. If $S$ is a curve, the Kawamata subadjunction theorem (see Theorem [2.8, [28, Theorem 1]) implies that $S$ is smooth, and we proceed with applying the Nadel-Shokurov vanishing theorem, the Riemann-Roch theorem, the Clifford theorem (see Theorem [2.19, [22, Theorem 5.4], [2, Section III.1]), and the Castelnuovo bound (see Theorem 2.20, [22, Theorem 6.4], 2, Section III.2]). Using these, we obtain various restrictions on the number of irreducible components of $S$, the degree of the curve $S$, and the genus of the curve $S$. In many cases these restrictions appear to be incompatible with a faithful action of $\bar{G}$ on $X$. Namely, suppose that $X$ is one of the three threefolds from Theorem 1.24 and $\bar{G} \cong \mathrm{A}_{6}$. Then all possibilities for $S$ are ruled out by the above arguments, except for the cases when either $X$ is the Segre cubic and $S$ is its singular point, or when $X \cong \mathbb{P}^{3}$ and $S$ is line whose $\bar{G}$-orbit consists of 6 lines. In the former case the contradiction is obtained by brute force (see Lemma 5.7). So we have to deal with the case when $X \cong \mathbb{P}^{3}$ and $S$ is line whose $\bar{G}$-orbit consists of 6 lines. If $S$ is not a center of canonical singularities of the log pair $\left(\mathbb{P}^{3}, \lambda \mathcal{M}\right)$, then we use Lemma 2.17 to obtain a contradiction. However, we can not obtain a contradiction here in general, because $\mathbb{P}^{3}$ is not $\bar{G}$-birationally superrigid! Indeed, for every line $L \subset \mathbb{P}^{3}$ whose $\bar{G}$ orbit consists of 6 lines, there exists a non-biregular $\bar{G}$-equivariant birational involution $\mathbb{P}^{3} \rightarrow \mathbb{P}^{3}$ discovered by Todd in [45] that is undefined along every line in the $\bar{G}$-orbit of $L$ (see Lemma 4.5 for a description). There are 12 such lines in $\mathbb{P}^{3}$ in total. These 12 lines split in two $\bar{G}$-orbits, and each $\bar{G}$-orbit gives us a non-biregular $\bar{G}$-equivariant birational involution $\mathbb{P}^{3} \rightarrow \mathbb{P}^{3}$, which we denote by $\iota$ and $\iota^{\prime}$, respectively. Applying $\iota$ and $\iota^{\prime}$ to $\mathcal{M}$ if necessary, we may assume that lines whose $\bar{G}$-orbit consist of 6 lines are not centers of canonical singularities of the log pair $(X, \lambda \mathcal{M})$ (see Lemma 4.7). Combining this with what we have already proved, we see that the singularities of the log pair $(X, \lambda \mathcal{M})$ are canonical up to the action of $\left\langle\bar{G}, \iota, \iota^{\prime}\right\rangle$, which implies that $\mathbb{P}^{3}$ is $\bar{G}$-birationally rigid and $\operatorname{Bir}^{\bar{G}}\left(\mathbb{P}^{3}\right)=\left\langle\operatorname{Aut}{ }^{\bar{G}}\left(\mathbb{P}^{3}\right), \iota, \iota^{\prime}\right\rangle$. Finally, we observe that

$$
\operatorname{Aut}^{\bar{G}}\left(\mathbb{P}^{3}\right) \cong\langle\bar{G}, \iota\rangle \cong\left\langle\bar{G}, \iota^{\prime}\right\rangle \cong \mathrm{S}_{6}
$$

and use this to prove that $\left\langle\operatorname{Aut}^{\bar{G}}\left(\mathbb{P}^{3}\right), \iota, \iota^{\prime}\right\rangle$ is the free product of two copies of $\mathrm{S}_{6}$ with amalgamated subgroup $\mathrm{A}_{6}$. Let us illustrate the described strategy by proving

Theorem 1.27. Suppose that $\bar{G}$ is one of the following subgroups: $\mathrm{A}_{7}, \mathrm{SL}_{2}\left(\mathbb{F}_{8}\right), \mathrm{PSp}_{4}\left(\mathbb{F}_{3}\right)$. Then $V$ is $\bar{G}$-birationally superrigid. 
Proof. If $V \cong \mathbb{P}^{3}$, then either $\bar{G} \cong \mathrm{A}_{7}$, or $\bar{G} \cong \mathrm{PSp}_{4}\left(\mathbb{F}_{3}\right)$, and the proof of Theorem 1.24 implies that $V$ is $\bar{G}$-birationally superrigid, since $\mathrm{A}_{7}$ and $\mathrm{PSp}_{4}\left(\mathbb{F}_{3}\right)$ contain subgroups isomorphic to $\mathrm{A}_{6}$. In fact, the $\bar{G}$-birational superrigidity of the threefold $V$ in the latter case is much easier to prove than Theorem 1.24, but we leave the details to the reader.

It follows from Theorem 1.16 and Corollary 1.22 that we may assume that $\bar{G} \cong \mathrm{SL}_{2}\left(\mathbb{F}_{8}\right)$ and $V$ is the threefold constructed in Example 1.14. Suppose that $V$ is not $\bar{G}$-birationally superrigid. Then it follows from [12, Theorem 4.2] or [7, Theorem 1.4.1] that there is a (non-empty) $\bar{G}$ invariant linear system $\mathcal{M}$ on $V$ such that $\mathcal{M}$ does not have fixed components, and $(V, \lambda \mathcal{M})$ is not canonical, where $\lambda$ is a positive rational number such that $\lambda \mathcal{M} \sim_{\mathbb{Q}}-K_{V}$. By Corollary 2.3 there is $\mu \in \mathbb{Q}$ such that $\mu<2 \lambda$ and $(V, \mu \mathcal{M})$ is $\log$ canonical and not Kawamata log terminal (see [29, Definition 3.5]).

Let $S$ be a subvariety in $V$ that is a minimal center of log canonical singularities of the $\log$ pair $(V, \mu \mathcal{M})$ (see [27], 28], [10, Definition 2.8]), and let $Z$ be the $\bar{G}$-orbit of the subvariety $S$. Then $S$ is either a point or a curve, since $\mathcal{M}$ does not have fixed components. Moreover, the subvariety $Z \subset V$ is a disjoint union of its components (see Lemma 2.7, [27, Proposition 1.5]).

Arguing as in the proof of [27, Theorem 1.10] or [28, Theorem 1], we can construct a $\bar{G}$ invariant effective $\mathbb{Q}$-divisor $D$ on $V$ such that $D \sim_{\mathbb{Q}}-\delta K_{V}$ for some positive rational number $\delta<2$, the log pair $(V, D)$ is $\log$ canonical, the subvariety $S$ is a minimal center of $\log$ canonical singularities of the log pair $(V, D)$, and the only centers of log canonical singularities (see [27, Definition 1.3]) of the log pair $(V, D)$ are irreducible components of $Z$ (see Corollary $[2.3$ and Lemma 2.13).

Let $\mathcal{I}_{Z}$ be its ideal sheaf. Put $q=h^{0}\left(\mathcal{O}_{V}\left(-K_{V}\right) \otimes \mathcal{I}_{Z}\right)=0$. Then

$$
h^{0}\left(\mathcal{O}_{Z} \otimes \mathcal{O}_{V}\left(-K_{V}\right)\right)=h^{0}\left(\mathcal{O}_{V}\left(-K_{V}\right)\right)-q=9-q \leqslant 9
$$

by the Nadel-Shokurov vanishing theorem (see Theorem 2.4, [30, Theorem 9.4.8]) and the Riemann-Roch theorem. Let $r$ be the number of irreducible components of the curve $Z$. Then $r \notin\{2,3, \ldots, 8\}$, since $\bar{G}$ can not act transitively on a finite set consisting of at least 2 and at most 8 elements (see [15]). Note that $Z=S$ if $r=1$.

Suppose that $S$ is a point. Then either $Z=S$ or $|Z|=r=9$ by (1.28). If $Z=S$, then $\bar{G}$ must act faithfully on the tangent space to $V$ at the point $S$, which is impossible, since $\mathrm{SL}_{2}\left(\mathbb{F}_{8}\right)$ has no non-trivial three-dimensional representations (see [15]). Thus, we see that $|Z|=9$. Let $F_{56}$ be the stabilizer subgroup in $\bar{G}$ of the point $S$. Then $\left|F_{56}\right|=|\bar{G}| / 9=56$, and hence one has $F_{56} \cong \mathbb{Z}_{2}^{3}: \mathbb{Z}_{7}$ (see [15]). On the other hand, the group $F_{56}$ must faithfully act on the tangent space to $V$ at the point $S$, which is impossible, since $\mathbb{Z}_{2}^{3}: \mathbb{Z}_{7}$ has no faithful three-dimensional representations.

Thus, we see that $S$ is a curve. Put $d=-K_{V} \cdot C$. By the Kawamata subadjunction theorem (see Theorem 2.8, [28, Theorem 1]), the curve $S$ is a smooth curve of genus $g$ such that $d>2 g-2$. In particular, the divisor $-\left.K_{V}\right|_{S}$ is not special. Thus, it follows from (1.28) and the RiemannRoch theorem that

$$
9-q=r(d-g+1)
$$

where either $r=1$, or $r=9$. If $r=9$, then it follows from (1.29) that $d=g$ and $q=0$, which is impossible, since $d>2 g-2$. Thus, we see that $r=1$, which simply means that $Z=S$. Then $\bar{G}$ acts faithfully on $S$, since $\bar{G}$ is simple and there are no points in $V$ that are $\bar{G}$-invariant. Moreover, it follows from (1.29) that $d=8+g-q$. Keeping in mind that $d>2 g-2$, we see that $g<10$. Then $g=7$ by the refined Hurwitz bound (see [6, Theorem 3.17], Theorem [2.18). In particular, we see that $d=15-q$, since $d=8+g-q$. 
Let $M_{1}$ and $M_{2}$ be general surfaces in $\mathcal{M}$, and let $H$ be a general surface in $\left|-K_{V}\right|$. Then it follows from [13, Theorem 3.1] that $\operatorname{mult}_{S}\left(M_{1} \cdot M_{2}\right) \geqslant 4 / \mu^{2}$, where $\mu<2 \lambda$. Thus, one has

$$
\frac{12}{\lambda^{2}}=M_{1} \cdot M_{2} \cdot H \geqslant \sum_{O \in H \cap S} \operatorname{mult}_{S}\left(M_{1} \cdot M_{2}\right) \geqslant \frac{4|H \cap S|}{\mu^{2}}>\frac{|H \cap S|}{\lambda^{2}},
$$

which implies that $|H \cap S|<12$. But $|H \cap S|=d=15-q$, which implies that $q \geqslant 4$. The latter is impossible, since $\mathrm{SL}_{2}\left(\mathbb{F}_{8}\right)$ has no non-trivial representations of dimension at most 6 .

The reader may find it useful to compare the proof of Theorem 1.20 (that uses a classical approach to birational rigidity) with the proof of Theorem 1.27 (that uses our new approach). Note that the proof of Theorem 1.27 does not use any information about the geometry of the variety $V$ acted on by the group $\mathrm{SL}_{2}\left(\mathbb{F}_{8}\right)$. Applying Theorem 1.7 and Corollary 1.12 and using classification of primitive subgroups in $\mathrm{SL}_{4}(\mathbb{C})$ and $\mathrm{SL}_{5}(\mathbb{C})$ (see [17]), we obtain

Corollary 1.30. Let $\bar{G}$ be a subgroup in $\mathrm{Cr}_{3}(\mathbb{C})$ that is isomorphic to either $\mathrm{A}_{7}$, or $\mathrm{PSp}_{4}\left(\mathbb{F}_{3}\right)$. If $\bar{G} \cong \mathrm{A}_{7}$, then its normalizer in $\mathrm{Cr}_{3}(\mathbb{C})$ is isomorphic to $\mathrm{S}_{7}$. If $\bar{G} \cong \mathrm{PSp}_{4}\left(\mathbb{F}_{3}\right)$, then its normalizer in $\mathrm{Cr}_{3}(\mathbb{C})$ is $\bar{G}$ itself.

The plan of the paper is as follows. In Section 2 we collect the well-known preliminary results on the log canonical singularities, make some remarks on varieties with group action and recall some general classical theorems. In Section 3 we collect the necessary facts about the structure of the groups $\mathrm{A}_{6}$ and $2 . \mathrm{A}_{6}$ and the relevant representation theory. In Sections 4 , 5 and 6 we study the projective space $\mathbb{P}^{3}$, the Segre cubic and the smooth quadric threefold according to the plan explained above, proving Theorems 4.8, 4.9, 5.5 and 6.2, respectively. These four theorems imply Theorem 1.24. In Appendix \$, we answer (negatively) a question on the existence of equivariant birational maps between two certain remarkable threefolds acted on by the group $\bar{\Gamma} \cong \mathrm{PSL}_{2}\left(\mathbb{F}_{11}\right)$ posed in [38, Remark 2.10]. Appendix B, written by Yu. Prokhorov, classifies terminal threefolds $X$ acted on by the group $\bar{G} \cong \mathrm{A}_{6}$ such that $X$ admits a $\bar{G}$-Mori fibration $\pi: X \rightarrow \mathbb{P}^{1}$.

We would like to thank T. Dokchitser, I. Dolgachev, Yu. Prokhorov, L. Rybnikov and E. Smirnov for very useful discussions and comments. We would like to thank the referee for many valuable and useful comments that helped us to improve the paper significantly.

\section{Preliminaries}

Throughout the paper we use the standard language of the singularities of pairs (see [29] and [14, Section 6]). By strictly log canonical singularities we mean log canonical singularities that are not Kawamata log terminal (see [29, Definition 3.5]).

Let $X$ be a variety with at most Kawamata log terminal singularities (see [29, Definition 3.5]), let $B_{X}$ be a formal linear combinations with non-negative rational coefficients of non-empty (but not necessarily complete) linear systems on $X$. Then we may write $B_{X}=\sum_{i=1}^{r} a_{i} B_{i}$, where $a_{i} \in \mathbb{Q}$, and $B_{i}$ is either a prime Weil divisor on the variety $X$, or a linear system without fixed components. We assume for simplicity that $B_{i} \neq B_{j}$ if $i \neq j$.

Remark 2.1. Usually $B_{X}$ is assumed to be an effective $\mathbb{Q}$-divisor. But sometimes it is necessary to assume that some or all components of $B_{X}$ are linear systems without fixed components (see [14, Section 6]). We will need such log pair throughout the paper, but do not want to stress on using them, since all properties of standard log pairs we plan to use hold for these generalized log pairs (see [29, Definition 4.6] and [14, Definition 6.16]). Moreover, we can always treat $B_{X}$ as a $\mathbb{Q}$-divisor by replacing each its mobile component by a weighted sum of its sufficiently general members (see [29, Theorem 4.8]). 
Suppose that $K_{X}+B_{X}$ is $\mathbb{Q}$-Cartier. Let $\pi: \bar{X} \rightarrow X$ be a birational morphism such that $\bar{X}$ is smooth. Then

$$
K_{\bar{X}}+\sum_{i=1}^{r} a_{i} \bar{B}_{i} \sim_{\mathbb{Q}} \pi^{*}\left(K_{X}+B_{X}\right)+\sum_{i=1}^{m} d_{i} E_{i},
$$

where $\bar{B}_{i}$ is the proper transforms of $B_{i}$ on the variety $\bar{X}$, and $E_{i}$ is an exceptional divisor of the morphism $\pi$, and $d_{i}$ is a rational number. We may assume that $\sum_{i=1}^{r} \bar{B}_{i}+\sum_{i=1}^{m} E_{i}$ is a divisor with simple normal crossing and every mobile linear system among $\bar{B}_{1}, \ldots, \bar{B}_{r}$ (if any) is free from base points.

Lemma 2.2. Suppose that $X$ has terminal singularities. Let $n$ be a positive integer such that $n K_{X}$ is a Cartier divisor. Then $\left(X,(1+n) \lambda B_{X}\right)$ is not log canonical if $\left(X, \lambda B_{X}\right)$ is not canonical.

Proof. There are positive rational numbers $c_{1}, \ldots, c_{r}$ such that $K_{\bar{X}} \sim_{\mathbb{Q}} \pi^{*}\left(K_{X}\right)+\sum_{i=1}^{m} c_{i} E_{i}$, because $X$ has terminal singularities and, in particular, $K_{X}$ is a $\mathbb{Q}$-Cartier divisor. Moreover, the numbers $n c_{1}, \ldots, n c_{r}$ are positive integers, since $n K_{X}$ is a Cartier divisor. On the other hand, we have

$$
\sum_{i=1}^{r} a_{i} \bar{B}_{i} \sim_{\mathbb{Q}} \pi^{*}\left(B_{X}\right)-\sum_{i=1}^{m} m_{i} E_{i}
$$

for some non-negative rational numbers $m_{1}, \ldots, m_{r}$. Then $d_{i}=c_{i}-m_{i}$ for every $i \in\{1, \ldots, r\}$.

Suppose that $\left(X, \lambda B_{X}\right)$ is not canonical. Then there exists $s \in\{1, \ldots, m\}$ such that $c_{s}-m_{s}=$ $d_{s}<0$ (see [14, Definition 6.16]). Thus, we have

$$
c_{s}-(1+n) m_{s}=c_{s}-m_{s}-n m_{s}<-n m_{s}<-1,
$$

because $m_{s}>c_{s} \geqslant 1 / n$, since $n c_{s}$ is a positive integer. On the other hand, we have

$$
K_{\bar{X}}+(1+n) \sum_{i=1}^{r} a_{i} \bar{B}_{i} \sim_{\mathbb{Q}} \pi^{*}\left(K_{X}+(1+n) B_{X}\right)+\sum_{i=1}^{m}\left(c_{i}-(1+n) m_{i}\right) E_{i},
$$

where $c_{s}-(1+n) m_{s}<-1$. Therefore, the log pair $\left(X,(1+n) \lambda B_{X}\right)$ is not log canonical.

Corollary 2.3. Suppose that $X$ has terminal Gorenstein singularities and $\left(X, \lambda B_{X}\right)$ is not canonical. Then $\left(X, 2 \lambda B_{X}\right)$ is not $\log$ canonical.

Suppose that $\left(X, B_{X}\right)$ is $\log$ canonical. Put $\hat{a}_{i}=a_{i}$ if $B_{i}$ is a prime Weil divisor, and $\hat{a}_{i}=0$ if $B_{i}$ is a linear system without fixed components. Put $\mathcal{I}\left(X, B_{X}\right)=\pi_{*}\left(\sum_{i=1}^{m}\left\lceil d_{i}\right\rceil E_{i}-\right.$ $\left.\sum_{i=1}^{r}\left\lfloor\hat{a}_{i}\right\rfloor \bar{B}_{i}\right)$.

Theorem 2.4 ([30, Theorem 9.4.8]). Let $H$ be a nef and big $\mathbb{Q}$-divisor on $X$, and let $D$ be a Cartier divisor on $X$ such that $D \equiv K_{X}+B_{X}+H$. Then $h^{i}\left(\mathcal{I}\left(X, B_{X}\right) \otimes D\right)=0$ for every $i \geqslant 1$.

Let $\mathcal{L}\left(X, B_{X}\right)$ be a subscheme of $X$ that corresponds to the ideal sheaf $\mathcal{I}\left(X, B_{X}\right)$. Put $\operatorname{LCS}\left(X, B_{X}\right)=\operatorname{Supp}\left(\mathcal{L}\left(X, B_{X}\right)\right)$. Note that $\mathcal{L}\left(X, B_{X}\right)$ is reduced, because $\left(X, B_{X}\right)$ is log canonical.

Theorem 2.5 ([41, Lemma 5.7], [27, Theorem 1.4]). Let $\zeta: X \rightarrow Z$ be a surjective morphism with connected fibers, and let $F$ be a fiber of $\zeta$. Then the locus $\operatorname{LCS}\left(X, B_{X}\right) \cap F$ is connected if $-\left(K_{X}+B_{X}\right)$ is $\eta$-nef and $\eta$-big.

Recall that there are standard names for $\mathcal{I}\left(X, B_{X}\right), \mathcal{L}\left(X, B_{X}\right)$ and $\operatorname{LCS}\left(X, B_{X}\right)$. Namely, the ideal $\mathcal{I}\left(X, B_{X}\right)$ is known as the multiplier ideal sheaf (see [30, Section 9.2]), the subscheme $\mathcal{L}\left(X, B_{X}\right)$ is known as the log canonical singularities subscheme (see [7, Definition 1.7.5]), and the locus $\operatorname{LCS}\left(X, B_{X}\right)$ is known as the locus of log canonical singularities (see [41, Definition 3.14]). 
Theorem 2.6 ([14, Theorem 6.29]). Suppose that $a_{1}=1$, and $B_{1}$ is a prime Weil divisor. Suppose that $B_{1}$ is a Cartier divisor on $X$, and $B_{1}$ is a normal variety that has at most Kawamata $\log$ terminal singularities. Then $\left(X, B_{X}\right)$ is $\log$ canonical along $B_{1}$ if and only if the $\log$ pair $\left(B_{1},\left.\sum_{i=2}^{r} a_{i} B_{i}\right|_{B_{1}}\right)$ is log canonical.

Let $Z$ be a center of $\log$ canonical singularities of the log pair $\left(X, B_{X}\right)$ (see [27, Definition 1.3]), and let $\mathbb{L} \mathbb{C} \mathbb{S}\left(X, B_{X}\right)$ be the set of all centers of log canonical singularities of the log pair $\left(X, B_{X}\right)$.

Lemma 2.7 ([27, Proposition 1.5]). Let $Z$ and $Z^{\prime}$ be elements of the set $\mathbb{L} \mathbb{C} \mathbb{S}\left(X, B_{X}\right)$, and let $Y$ be any irreducible reduced component of $Z \cdot Z^{\prime}$. Then $Y \in \mathbb{L} \mathbb{C} S\left(X, B_{X}\right)$.

Suppose that $Z$ is a minimal center in $\mathbb{L} \mathbb{C S}\left(X, B_{X}\right)$ (see [27], 28, [10, Definition 2.8]).

Theorem 2.8 ([28, Theorem 1$])$. Let $\Delta$ be an ample $\mathbb{Q}$-Cartier $\mathbb{Q}$-divisor on $X$. Then

- the variety $Z$ is normal and has at most rational singularities,

- there exists an effective $\mathbb{Q}$-divisor $B_{Z}$ on $Z$ such that $\left.\left(K_{X}+B_{X}+\Delta\right)\right|_{Z} \sim_{\mathbb{Q}} K_{Z}+B_{Z}$, and $\left(Z, B_{Z}\right)$ has Kawamata log terminal singularities.

Let $\bar{G} \subseteq \operatorname{Aut}(X)$ be a finite subgroup.

Lemma 2.9. Suppose that $X$ is a curve. Let $\Sigma \subset X$ be a $\bar{G}$-orbit. If $|\Sigma|=1$, then $\bar{G}$ is cyclic. If $\bar{G} \cong \mathrm{A}_{5}$, then $|\Sigma| \in\{12,20,30,60\}$. If $\bar{G} \cong \mathrm{A}_{6}$, then $|\Sigma| \in\{60,72,90,120,180,360\}$.

Proof. If $\Sigma$ is a point, then $\bar{G}$ acts faithfully on the tangent space to $X$ at the point $\Sigma$.

Lemma 2.10. Suppose that $X$ is a curve of genus $g$ and $\bar{G} \cong \mathrm{A}_{6}$. If $g \leqslant 34$, then $g \in$ $\{10,16,19,25,31\}$. If $g=10$, then $X$ does not contain $\bar{G}$-orbits of length 120 .

Proof. Let $\bar{F} \subset \bar{G}$ be a stabilizer of a point in $X$. Then $\bar{F} \cong \mathbb{Z}_{k}$ for some $k \in\{1,2,3,4,5,6\}$ by Lemma 2.9. Put $\bar{X}=X / \bar{G}$. Then $\bar{X}$ is a smooth curve of genus $\bar{g}$. The Riemann-Hurwitz formula gives

$$
2 g-2=360(2 \bar{g}-2)+180 a_{2}+240 a_{3}+270 a_{4}+288 a_{5}+300 a_{6},
$$

where $a_{k}$ is the number of $\bar{G}$-orbits in $X$ with a stabilizer of a point isomorphic to $\mathbb{Z}_{k}$.

Suppose that $g \leqslant 34$. Note that $g \neq 0$ by the classification of finite subgroups of $\mathrm{PGL}_{2}(\mathbb{C})$, and $g \neq 1$ since $\bar{G}$ is non-solvable. Since $a_{k} \geqslant 0$, one has $\bar{g}=0$, and

$$
2 g-2=-720+180 a_{2}+240 a_{3}+270 a_{4}+288 a_{5}+300 a_{6}
$$

which implies that $g \in\{10,16,19,25,31\}$. The only solution to (2.11) for $g=10$ is $\left(a_{2}, a_{3}, a_{4}, a_{5}, a_{6}\right)=(1,0,1,1,0)$, which completes the proof.

Remark 2.12. We do not claim that every case listed in Lemma 2.10 is realized. The obtained restrictions on the genus are enough for our purposes.

Suppose, in addition, that $B_{X}$ is $\bar{G}$-invariant. Then $g(Z) \in \mathbb{L} \mathbb{C} S\left(X, B_{X}\right)$ for every $g \in \bar{G}$, and the locus $\operatorname{LCS}\left(X, B_{X}\right)$ is $\bar{G}$-invariant. It follows from Lemma 2.7 that $Z \cap g(Z) \neq \varnothing$ if and only if $Z=g(Z)$ for every $g \in \bar{G}$, because $Z$ is a minimal center in $\mathbb{L} \mathbb{C} \mathbb{S}\left(X, B_{X}\right)$.

Lemma 2.13. Suppose that the divisor $B_{X}$ is ample. Let $\epsilon$ be an arbitrary rational number such that $\epsilon>0$. Then there exists an effective $\bar{G}$-invariant $\mathbb{Q}$-divisor $D$ on the variety $X$ such that the set $\mathbb{L} \mathbb{C S}(X, D)$ consists of all components of the $\bar{G}$-orbit of $Z$, the log pair $(X, D)$ is $\log$ canonical, and the equivalence $D \sim_{\mathbb{Q}}(1+\epsilon) B_{X}$ holds.

Proof. See the proofs of [27, Theorem 1.10], [28, Theorem 1], [10, Lemma 2.11].

Suppose, in addition, that $X$ is a Fano variety. Put

$$
\operatorname{lct}(X, \bar{G})=\sup \left\{\begin{array}{l|l}
\lambda \in \mathbb{Q} & \begin{array}{l}
\text { the log pair }(X, \lambda D) \text { has log canonical singularities } \\
\text { for every } \bar{G} \text {-invariant effective } \mathbb{Q} \text {-divisor } D \sim_{\mathbb{Q}}-K_{X}
\end{array}
\end{array}\right\} \in \mathbb{R} \text {. }
$$


Remark 2.14. If $X$ is smooth, then it follows from [9, Theorem A.3] that $\operatorname{lct}(X, \bar{G})=\alpha_{\bar{G}}(X)$, where $\alpha_{\bar{G}}(X)$ is the $\bar{G}$-invariant $\alpha$-invariant of the variety $X$ introduced in [43] and [44].

Suppose that $X \cong \mathbb{P}^{1} \times \mathbb{P}^{1}$.

Lemma 2.15. Suppose that $\bar{G} \cong \mathrm{A}_{5}$. Let $\Sigma \subset X$ be a $\bar{G}$-invariant subset. Then $|\Sigma| \geqslant 12$.

Proof. The required assertion follows from Lemma 2.9.

Let $L_{1}$ and $L_{2}$ be fibers of the projection to the first and the second factor, respectively.

Lemma 2.16. The inequality $\operatorname{lct}(X, \bar{G}) \geqslant 1$ holds if and only if the linear systems $\left|L_{1}\right|,\left|L_{2}\right|$, $\left|L_{1}+L_{2}\right|$ contain no $\bar{G}$-invariant curves.

Proof. If there is a $\bar{G}$-invariant curve in $\left|L_{1}\right|$ or $\left|L_{2}\right|$ or $\left|L_{1}+L_{2}\right|$, then $\operatorname{lct}(X, \bar{G})=1 / 2$ (see [8, Theorem 1.7]).

Suppose that $\left|L_{1}\right|,\left|L_{2}\right|,\left|L_{1}+L_{2}\right|$ contain no $\bar{G}$-invariant curves, but $\operatorname{lct}(X, \bar{G})<1$. There are $\lambda \in \mathbb{Q}$ and an effective $\bar{G}$-invariant $\mathbb{Q}$-divisor $D$ on $X$ such that $\lambda<1$ and $D \sim_{\mathbb{Q}} 2\left(L_{1}+L_{2}\right)$, but $(X, \lambda D)$ is not Kawamata log terminal. We may assume that $(X, \lambda D)$ is $\log$ canonical.

Suppose that there is a $\bar{G}$-invariant curve $C \subset X$ such that $\lambda D=\mu C+\Omega$, where $\mu \geqslant 1$, and $\Omega$ is an effective $\bar{G}$-invariant $\mathbb{Q}$-divisor, whose support does not contain any component of the curve $C$. Then $C \sim a L_{1}+b L_{2}$ for some non-negative integers $a$ and $b$. Then either $a \geqslant 2$ or $b \geqslant 2$. But

$$
2>2 \lambda=\lambda\left(D \cdot L_{1}\right)=(\mu C+\Omega) \cdot L_{1} \geqslant C \cdot L_{1}=b,
$$

which implies that $b \leqslant 1$. Similarly, we see that $a=C \cdot L_{2} \leqslant 1$, which is a contradiction.

We see that the locus $\operatorname{LCS}(X, \lambda D)$ is a finite $\bar{G}$-invariant set. Thus, the locus $\operatorname{LCS}(X, \lambda D)$ consists of a single point $O \in X$ by Theorem 2.5. Let $H$ be the unique curve in $\left|L_{1}+L_{2}\right|$ that is singular at $O$. Then $H$ must be $\bar{G}$-invariant, which is a contradiction, because the linear system $\left|L_{1}+L_{2}\right|$ contains no $\bar{G}$-invariant divisors.

Let us identify $X \cong \mathbb{P}^{1} \times \mathbb{P}^{1}$ with a smooth quadric surface in $\mathbb{P}^{3}$, and let us identify $\bar{G}$ with a subgroup in $\operatorname{Aut}\left(\mathbb{P}^{3}\right)$ such that $X$ is $\bar{G}$-invariant. Let $\phi: \mathrm{SL}_{4}(\mathbb{C}) \rightarrow \operatorname{Aut}\left(\mathbb{P}^{3}\right)$ be the natural projection. Then there is a finite subgroup $G \subset \mathrm{SL}_{4}(\mathbb{C})$ such that $\bar{G}=\phi(G)$. Put $W=\mathbb{C}^{4}$.

Lemma 2.17. Suppose that $\bar{G} \cong G \cong \mathrm{A}_{5}$, and $W \cong \mathbb{C}^{4}$ is an irreducible representation of the group $G$. Assume that $B_{X} \sim_{\mathbb{Q}} m L_{1}+(8-m) L_{2}$, where $m$ is a rational number such that $1<m<2$. Then the locus $\operatorname{LCS}\left(X, B_{X}\right)$ consists of finitely many points.

Proof. Let $U$ be a two-dimensional representation of the group 2. $\mathrm{A}_{5}$. Then the center of the group $2 . \mathrm{A}_{5}$ acts trivially on $U \otimes U^{\vee}$, and $U \otimes U^{\vee} \cong W$ as representations of $\mathrm{A}_{5}$. Therefore, one has

$$
H^{0}\left(X, \mathcal{O}_{X}\left(L_{1}+n L_{2}\right)\right) \cong U \otimes \operatorname{Sym}^{n}\left(U^{\vee}\right)
$$

as representations of $\mathrm{A}_{5}$.

Suppose that the locus $\operatorname{LCS}\left(X, B_{X}\right)$ does not consist of finitely many points. Then there is a $\bar{G}$-invariant curve $C \subset X$ such that $B_{X}=\mu C+\Omega$, where $\mu \geqslant 1$ and $\Omega$ is an effective $\bar{G}$-invariant $\mathbb{Q}$-divisor, whose support does not contain any component of the curve $C$. Then $C \sim a L_{1}+b L_{2}$ for some non-negative integers $a$ and $b$. Then either $a \geqslant 2$ or $b \geqslant 2$, since the linear systems $\left|L_{1}\right|,\left|L_{2}\right|$ and $\left|L_{1}+L_{2}\right|$ do not contain $\bar{G}$ invariant divisors (indeed, $W$ is an irreducible $\bar{G}$ representations, so that $\mathbb{P}(W)$ does not contain $\bar{G}$-invariant hyperplanes, and both $U$ and $U^{\vee}$ are irreducible $G$-representations, so that $\mathbb{P}(U) \cong \mathbb{P}^{1}$ and $\mathbb{P}\left(U^{\vee}\right) \cong \mathbb{P}^{1}$ do not contain $\bar{G}$-invariant points). On the other hand

$$
2>m=B_{X} \cdot L_{2}=(\mu C+\Omega) \cdot L_{2} \geqslant C \cdot L_{2}=a,
$$


which implies that either $a=0$ or $a=1$. Similarly, we see that

$$
7>8-m=B_{X} \cdot L_{1}=(\mu C+\Omega) \cdot L_{1} \geqslant C \cdot L_{1}=b,
$$

which implies that $b \leqslant 6$. If $a=0$, then $C \in\left|b L_{2}\right|$, and thus $C$ is $\bar{G}$-invariant. The latter is implied by Lemma 2.9 (actually, Lemma 2.9 shows that the linear system $\left|b L_{2}\right|$ does not contain $\bar{G}$-invariant curves for $b \leqslant 11$ ). If $a=1$, then $C \in\left|L_{1}+b L_{2}\right|$. Now a direct computation 11 shows that $U \otimes \operatorname{Sym}^{n}\left(U^{\vee}\right)$ does not contain one-dimensional $G$-invariant subspaces for $b \leqslant 6$. The obtained contradiction completes the proof.

Let $C$ be a smooth irreducible curve of genus $g$.

Theorem 2.18. Suppose that $g \geqslant 2$. Then

$$
|\operatorname{Aut}(C)| \leqslant\left\{\begin{array}{l}
320 \text { if } g=9, \\
432 \text { if } g=10 \\
240 \text { if } g=11 \\
120 \text { if } g=12, \\
360 \text { if } g=13, \\
504 \text { if } g=15
\end{array}\right.
$$

and the inequality $|\operatorname{Aut}(C)| \leqslant 84(g-1)$ holds for any $g \geqslant 2$.

Proof. The inequality $|\operatorname{Aut}(C)| \leqslant 84(g-1)$ is the famous Hurwitz bound (see [6, Theorem 3.17]), the exact bounds for particular genera may be found in [6, Table 13].

Let $D$ be an effective divisor on the curve $C$.

Theorem 2.19 $\left(\left[22\right.\right.$, Theorem 5.4]). If $h^{1}\left(\mathcal{O}_{C}(D)\right) \neq 0$, then

$$
h^{0}\left(\mathcal{O}_{C}(D)\right) \leqslant \frac{\operatorname{deg}(D)}{2}+1 \text {. }
$$

Suppose that there is an embedding $\zeta: C \rightarrow \mathbb{P}^{n}$ such that $\zeta(C)$ is a curve of degree $d$.

Theorem 2.20 ([2, Section III.2]). If $\zeta(C)$ is not contained in any hyperplane in $\mathbb{P}^{n}$, then

$$
g \leqslant \frac{m(m-1)(n-1)}{2}+m \varepsilon,
$$

where $m=\lfloor(d-1) /(n-1)\rfloor$ and $\varepsilon=d-1-m(n-1)$.

\section{Alternating Group}

Let $\mathrm{S}_{6}$ be the group of permutations of the set $\{1,2,3,4,5,6\}$.

Definition 3.1. Let $H \subset \mathrm{A}_{6}$ be a subgroup that is isomorphic to $\mathrm{A}_{5}, \mathrm{~S}_{4}$ or $\mathrm{A}_{4}$. Then we say that the embedding $H \subset \mathrm{A}_{6}$ is standard if one of the following conditions is satisfied:

- if $H \cong \mathrm{A}_{5}$ and $H$ is conjugate to the subgroup of even permutations of $\{1,2,3,4,5\}$,

- if $H \cong \mathrm{S}_{4}$ and $H$ is conjugate to the subgroup of permutations of the set $\{1,2,3,4\}$ such that the odd permutations of $\{1,2,3,4\}$ are twisted by the transposition $(5,6)$,

- if $H \cong \mathrm{A}_{4}$ and $H$ is conjugate to the subgroup of even permutations of $\{1,2,3,4\}$.

We say that the embedding $H \subset \mathrm{A}_{6}$ is non-standard if it is not standard?

\footnotetext{
${ }^{1}$ We used the Magma software [5] to carry it out.

${ }^{2}$ Note that the non-standard and standard embeddings of the group $H$ into $\mathrm{A}_{6}$ are interchanged by Aut( $\left.\mathrm{A}_{6}\right)$.
} 
Let $2 . \mathrm{A}_{6}$ be the group such that there exists a non-splitting exact sequence of groups

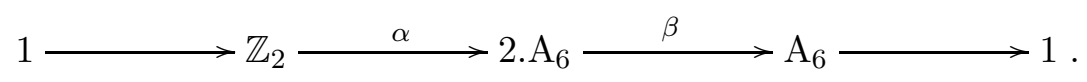

Note that $\alpha\left(\mathbb{Z}_{2}\right)$ is the center of the group $2 . \mathrm{A}_{6}$.

Definition 3.2. Let $H \subset 2 . \mathrm{A}_{6}$ such that $\beta(H)$ is isomorphic to $\mathrm{A}_{5}, \mathrm{~S}_{4}$ or $\mathrm{A}_{4}$. Then we say that the embedding $H \subset 2 . \mathrm{A}_{6}$ is standard if $\beta(H) \subset \mathrm{A}_{6}$ is standard, and we say that the embedding $H \subset 2 . \mathrm{A}_{6}$ is non-standard if $\beta(H) \subset \mathrm{A}_{6}$ is non-standard.

Let $\bar{G}$ be a finite subgroup in $\operatorname{Aut}\left(\mathbb{P}^{3}\right)$ such that $\bar{G} \cong \mathrm{A}_{6}$.

Lemma $3.3([15])$. Every maximal proper subgroup of the group $\bar{G} \cong \mathrm{A}_{6}$ is isomorphic to either to $\mathrm{A}_{5}$, or $\mathrm{S}_{4}$, or $\left(\mathbb{Z}_{3} \times \mathbb{Z}_{3}\right) \rtimes \mathbb{Z}_{4}$. Moreover, up to conjugation, the group $\bar{G}$ contains one subgroup isomorphic to $\left(\mathbb{Z}_{3} \times \mathbb{Z}_{3}\right) \rtimes \mathbb{Z}_{4}$, one subgroup isomorphic to $\left(\mathbb{Z}_{3} \times \mathbb{Z}_{3}\right) \rtimes \mathbb{Z}_{2}$, two subgroups isomorphic to $\mathrm{S}_{4}$ (respectively, $\mathrm{A}_{5}, \mathrm{~A}_{4}$ ).

Put $W=\mathbb{C}^{4}$. There exists a finite subgroup $G \subset \mathrm{SL}_{4}(\mathbb{C})$ such that $G \cong 2 . \mathrm{A}_{6}$ and $\bar{G}=$ $\phi(G) \subset \operatorname{Aut}\left(\mathbb{P}^{3}\right) \cong \mathrm{PGL}_{4}(\mathbb{C})$ where $\phi: \mathrm{SL}_{4}(\mathbb{C}) \rightarrow \operatorname{Aut}\left(\mathbb{P}^{3}\right)$ is the natural projection.

Remark 3.4. The group $G$ has two irreducible four-dimensional representations (see [15]), which implies that we may identify $W$ with one of them, because another one differs from $W$ by an outer automorphism of the group $G$. Thus, we may assume that the natural action of the group $G$ on $\Lambda^{2}(W) \cong \mathbb{C}^{6}$ arises from the permutation representation of the group $\bar{G} \cong \mathrm{A}_{6}$.

Let $\bar{F} \subset \bar{G}$ be a subgroup, and let $F \subset G$ be a subgroup such that $\phi(F)=\bar{F}$.

Remark 3.5. Suppose that $F \cong 2 . \mathrm{A}_{5} \subset 2 . \mathrm{A}_{6}$. Then

$$
\mathbb{C}^{4} \cong W \cong\left\{\begin{array}{l}
U \oplus U^{\prime} \text { if } F \subset G \text { is standard, } \\
\operatorname{Sym}^{3}(U) \cong \operatorname{Sym}^{3}\left(U^{\prime}\right) \text { if } F \subset G \text { is non-standard }
\end{array}\right.
$$

where $U$ and $U^{\prime}$ are different two-dimensional representations of the group $F \cong 2 . A_{5}$.

Lemma 3.6. Suppose that $F \cong 2 . A_{5}$ and $F \subset G$ is a non-standard embedding. Then there exists an irreducible $\bar{F}$-invariant smooth rational cubic curve $Z \subset \mathbb{P}^{3}$.

Proof. The required assertion follows from Remark 3.5.

Let $z$ and $e$ be the non-trivial element in the center of $G$ and the identity element, respectively.

Lemma 3.7 (cf. [10, Lemma 4.7]). Any semi-invariant of the group $G$ is its invariant. The group $G$ does not have invariants of odd degree, as well as invariants of degree at most 7 . On the other hand, the group $G$ has two linearly independent invariants of degree 8 .

Proof. Semi-invariants of the group $G$ are its invariants, because the center of the group $G$ is contained in its commutator, and the group $\bar{G}$ is a simple non-abelian group.

The group $G$ does not have invariants of odd degree, because $G$ contains a scalar matrix whose non-zero entries are -1 . Therefore, to prove that $G$ has no invariants of degree at most 7 , it is enough to show that $G$ does not have invariants of degree 4 and 6 .

Let $\chi_{m}$ be the character of the representation $\operatorname{Sym}^{m}(W)$ (cf. Remark 3.4). Put $\chi=\chi_{1}$.

The values of the characters $\chi, \chi_{4}, \chi_{6}$ and $\chi_{8}$ are listed in the following table: 


\begin{tabular}{|c|c|c|c|c|c|c|c|c|c|c|}
\hline & {$[5,1]_{10}$} & {$[5,1]_{5}$} & {$[4,2]_{8}$} & {$[3,3]_{6}$} & {$[3,3]_{3}$} & {$[3,1,1,1]_{6}$} & {$[3,1,1,1]_{3}$} & {$[2,2,1,1]_{4}$} & $z$ & $e$ \\
\hline$\#$ & 144 & 144 & 180 & 40 & 40 & 40 & 40 & 90 & 1 & 1 \\
\hline$\chi$ & 1 & -1 & 0 & -1 & 1 & 2 & -2 & 0 & -4 & 4 \\
\hline$\chi_{4}$ & 0 & 0 & -1 & 2 & 2 & -4 & -4 & 3 & 35 & 35 \\
\hline$\chi_{6}$ & -1 & -1 & 0 & 3 & 3 & 3 & 3 & -4 & 84 & 84 \\
\hline$\chi_{8}$ & 0 & 0 & 1 & 3 & 3 & 3 & 3 & 5 & 165 & 165 \\
\hline
\end{tabular}

where the first row lists the types of the elements in $G$ (for example, the symbol $[5,1]_{10}$ denotes the set 3 of elements of order 10 whose image in $A_{6}$ is a product of disjoint cycles of length 5 and 1 ).

Recall that there is a natural inner product $\langle\cdot, \cdot\rangle$ defined for the characters $\theta$ and $\theta^{\prime}$ by

$$
\left\langle\theta, \theta^{\prime}\right\rangle=\frac{1}{|G|} \sum_{g \in G} \theta(g) \overline{\theta^{\prime}(g)} .
$$

Let $\chi_{0}$ be the trivial character of $G$. Then $\left\langle\chi_{4}, \chi_{0}\right\rangle=\left\langle\chi_{6}, \chi_{0}\right\rangle=0$, so that $G$ has no invariants of degree 4 and 6 . On the other hand, $\left\langle\chi_{8}, \chi_{0}\right\rangle=2$, which means that the group $G$ has exactly two linearly independent invariants of degree 8.

Suppose that $\bar{F}$ is a stabilizer of a point $P \in \mathbb{P}^{3}$.

Lemma 3.8. Let $\Sigma \subset \mathbb{P}^{3}$ be the $\bar{G}$-orbit of the point $P \in \mathbb{P}^{3}$. Then $|\Sigma| \geqslant 36$.

Proof. It follows from Lemma 3.7 that $|\Sigma| \geqslant 8$ and $|\Sigma|$ is even. Suppose that $|\Sigma| \leqslant 35$. Then

$$
45=\frac{|\bar{G}|}{8} \geqslant|\bar{F}| \geqslant \frac{|\bar{G}|}{35}>10,
$$

which implies that $|\bar{F}| \in\{12,18,36\}$ by Lemma 3.3.

Let us consider the vector space $W$ as a representation of the group $F$, and let $\chi$ be its character. There is a homomorphism $\theta: F \rightarrow \mathbb{C}^{*}$ such that the inner product $\langle\theta, \chi\rangle \neq 0$.

Suppose that $|\bar{F}|=36$. Then $\bar{F} \cong\left(\mathbb{Z}_{3} \times \mathbb{Z}_{3}\right) \rtimes \mathbb{Z}_{4}$ by Lemma 3.3.

The structure of the group $F$ and the values of $\chi$ are given in the following table:

\begin{tabular}{|c|c|c|c|c|c|c|c|c|}
\hline & {$[4,2]_{8}$} & {$[3,3]_{6}$} & {$[3,3]_{3}$} & {$[3,1,1,1]_{6}$} & {$[3,1,1,1]_{3}$} & {$[2,2,1,1]_{4}$} & $z$ & $e$ \\
\hline$\#$ & 36 & 4 & 4 & 4 & 4 & 18 & 1 & 1 \\
\hline$\chi$ & 0 & -1 & 1 & 2 & -2 & 0 & -4 & 4 \\
\hline
\end{tabular}

where we use the notation that are used in the proof of Lemma 3.7 .

We have $[F, F] \cong 2$. $\left(\mathbb{Z}_{3} \times \mathbb{Z}_{3}\right)$, which gives $\theta(g)=1$ for any $g \in F$ that is not of type $[4,2]_{8}$ and $[2,2]_{4}$. Hence $\langle\theta, \chi\rangle=0$, which is a contradiction.

Suppose that $|\bar{F}|=18$. Then $\bar{F} \cong\left(\mathbb{Z}_{3} \times \mathbb{Z}_{3}\right) \rtimes \mathbb{Z}_{2}$ by Lemma 3.3. Arguing as above, we get $\langle\theta, \chi\rangle=0$.

Suppose that $|\bar{F}|=12$. Then $\bar{F} \cong \mathrm{A}_{4}$ by Lemma 3.3 .

Up to conjugation, the group $\bar{G}$ contains two subgroups isomorphic to $\mathrm{A}_{4}$.

If $\bar{F} \subset \bar{G}$ is a standard embedding, then the values of $\chi$ are given in the following table:

\begin{tabular}{|c|c|c|c|c|c|}
\hline & {$[3,1,1,1]_{6}$} & {$[3,1,1,1]_{3}$} & {$[2,2,1,1]_{4}$} & $z$ & $e$ \\
\hline$\#$ & 8 & 8 & 6 & 1 & 1 \\
\hline$\chi$ & 2 & -2 & 0 & -4 & 4 \\
\hline
\end{tabular}

If $\bar{F} \subset \bar{G}$ is a non-standard embedding, then the values of $\chi$ are given in the following table:

\footnotetext{
3 Note that these sets do not coincide with conjugacy classes. For example, the image of the set of the elements of type $[5,1]_{10}$ under the natural projection $2 . \mathrm{A}_{6} \rightarrow \mathrm{A}_{6}$ is a union of two different conjugacy classes in $\mathrm{A}_{6}$.
} 


\begin{tabular}{|c|c|c|c|c|c|}
\hline & {$[3,3]_{6}$} & {$[3,3]_{3}$} & {$[2,2,1,1]_{4}$} & $z$ & $e$ \\
\hline$\#$ & 8 & 8 & 6 & 1 & 1 \\
\hline$\chi$ & -1 & 1 & 0 & -4 & 4 \\
\hline
\end{tabular}

We have $[F, F] \cong 2 .\left(\mathbb{Z}_{2} \times \mathbb{Z}_{2}\right)$. So $\theta(g)=1$ for any $g \in F$ of order different from 3 and 6 , and $\theta(g)=\theta\left(g^{2}\right)$ for all $g \in F$ of order 6 . Now we can check that $\langle\theta, \chi\rangle=0$, which is a contradiction.

Lemma 3.9. Let $C$ be a smooth irreducible $\bar{G}$-invariant curve in $\mathbb{P}^{3}$ of genus $g \geqslant 13$, and let $\mathcal{I}_{C}$ be its ideal sheaf. Then $h^{0}\left(\mathcal{O}_{\mathbb{P}^{3}}(i) \otimes \mathcal{I}_{C}\right)=0$ for any $i \in\{1,2,3\}$.

Proof. It follows from Theorem 2.20 and Lemma 2.10 that $d>4$. Hence $h^{0}\left(\mathcal{O}_{\mathbb{P}^{3}}(2) \otimes \mathcal{I}_{C}\right)=0$, since $G$ does not have semi-invariants of degree 2 by Lemma 3.7 .

Suppose that there is a cubic surface $X \subset \mathbb{P}^{3}$ such that $C \subset X$. Then $h^{0}\left(\mathcal{O}_{\mathbb{P}^{3}}(3) \otimes \mathcal{I}_{C}\right) \geqslant 2$, since $G$ does not have semi-invariants of degree 3 by Lemma 3.7. Thus, there is a cubic surface $X^{\prime} \subset \mathbb{P}^{3}$ such that $C \subset X^{\prime} \neq X$. Thus $C \subset X \cap X^{\prime}$, and $X$ and $X^{\prime}$ are irreducible, because $C$ is contained neither in a quadric nor in a plane. We see that $d \leqslant 9$. Hence, we have $g \leqslant 12$ by Theorem 2.20, which is a contradiction.

\section{Projective Space}

Let $\bar{G}$ be a subgroup in $\operatorname{Aut}\left(\mathbb{P}^{3}\right)$ such that $\bar{G} \cong \mathrm{A}_{6}$. Then there is a subgroup $\hat{G} \subset \operatorname{Aut}\left(\mathbb{P}^{3}\right)$ such that $\bar{G} \subset \hat{G} \cong \mathrm{S}_{6}$, which implies that $\hat{G} \subseteq \operatorname{Aut}^{\bar{G}}\left(\mathbb{P}^{3}\right)$, because $\bar{G}$ is a normal subgroup of the group $\hat{G}$ (recall that $\operatorname{Aut}^{\bar{G}}\left(\mathbb{P}^{3}\right)$ is the normalizer of the group $\bar{G}$ in the group $\operatorname{Aut}\left(\mathbb{P}^{3}\right)$ ).

The main purpose of this section is to prove that $\mathbb{P}^{3}$ is $\bar{G}$-birationally rigid and to describe the group $\operatorname{Bir}^{\bar{G}}\left(\mathbb{P}^{3}\right)$ (see Theorem 1.24). We will do this in several steps. But first of all, we must study lines in $\mathbb{P}^{3}$ whose $\bar{G}$-orbits consists of 6 lines (there are 12 such lines in total, the groups $\hat{G}$ acts transitively on them, and they form two $\bar{G}$-orbits consisting of 6 lines each).

Let $\bar{G}_{1} \subset \bar{G}$ be a subgroup such that $\bar{G}_{1} \cong \mathrm{A}_{5}$ and the embedding $\mathrm{A}_{5} \cong \bar{G}_{1} \subset \bar{G} \cong \mathrm{A}_{6}$ is standard (see Definition 3.1). Then there are two disjoint $\bar{G}_{1}$-invariant lines $L_{1}$ and $L_{1}^{\prime}$ in $\mathbb{P}^{3}$ by Remark 3.5. Denote by $L_{1}, \ldots, L_{6}\left(L_{1}^{\prime}, \ldots, L_{6}^{\prime}\right.$, respectively) the lines in $\mathbb{P}^{3}$ that are the images of $L_{1}\left(L_{1}^{\prime}\right.$, respectively) under the action of $\bar{G}$. Put $\mathrm{L}=\left\{L_{1}, \ldots, L_{6}\right\}$ and $\mathrm{L}^{\prime}=\left\{L_{1}^{\prime}, \ldots, L_{6}^{\prime}\right\}$.

Lemma 4.1. The curve $\sum_{i=1}^{6} L_{i}+\sum_{i=1}^{6} L_{i}^{\prime}$ is a $\hat{G}$-orbit of the line $L_{1}$. The 12 lines of $\mathrm{L} \cup \mathrm{L}^{\prime}$ are pairwise disjoint. For any 4 lines among the lines $L_{1}, \ldots, L_{6}\left(L_{1}^{\prime}, \ldots, L_{6}^{\prime}\right.$, respectively), there are 2 lines in $\mathbb{P}^{3}$ that intersect them. There are no lines in $\mathbb{P}^{3}$ that intersect 5 lines among $L_{1}, \ldots, L_{6}\left(L_{1}^{\prime}, \ldots, L_{6}^{\prime}\right.$, respectively).

Proof. The first assertion of the lemma follows from the construction of the lines $L_{i}$ and $L_{i}^{\prime}$. To prove the second assertion note that the stabilizers of any two of the 12 lines of $\mathrm{L} \cup \mathrm{L}^{\prime}$ except for the case of the lines $L_{i}$ and $L_{i}^{\prime}$ corresponding to one and the same stabilizer $\mathrm{A}_{5} \subset \bar{G}$ (i. e. two standard subgroups isomorphic to $\mathrm{A}_{5}$ ) generate together the whole group $\bar{G} \cong \mathrm{A}_{6}$. In particular, all 12 lines of $L \cup L^{\prime}$ are pairwise distinct, since otherwise there would exist a $\bar{G}$-invariant line in $\mathbb{P}^{3}$. Similarly, if some two of the 12 lines of $L \cup L^{\prime}$ intersected at a point $P$, then there would exist a $\bar{G}$-invariant point in $\mathbb{P}^{3}$.

Suppose that there exist more than 2 lines in $\mathbb{P}^{3}$ that intersect some 4 of the lines of $\mathrm{L}$ (say, $L_{1}$, $L_{2}, L_{3}$ and $L_{4}$ ). Then the lines $L_{1}, \ldots, L_{4}$ are contained in a (unique) smooth quadric $X \subset \mathbb{P}^{3}$. Note that there is an element $g \in \bar{G} \cong \mathrm{A}_{6}$ that preserves the lines $L_{1}$ and $L_{6}$, interchanges the lines $L_{2}$ and $L_{3}$ and interchanges the lines $L_{4}$ and $L_{5}$. The quadric $X$ is invariant under $g$ (indeed, an intersection of two distinct smooth quadrics in $\mathbb{P}^{3}$ cannot contain three skew lines). Hence $X$ contains the line $L_{5}$. Similarly, $X$ contains the line $L_{6}$, and thus $X$ is $\bar{G}$-invariant. The latter contradicts Lemma 3.7. Therefore, to prove the third assetion of the lemma we may 
suppose that there exist exactly 1 line $L_{1234}$ in $\mathbb{P}^{3}$ that intersects the lines $L_{1}, \ldots, L_{4}$. Note that the group $\bar{G}$ acts transitively on the 4 -tuples of the lines of $\mathrm{L}$. Thus for any 4 lines $L_{i_{1}}, \ldots, L_{i_{4}}$ there exists a unique line $L_{i_{1} \ldots i_{4}}$ that intersects $L_{i_{1}}, \ldots, L_{i_{4}}$. Put $P_{i_{2} i_{3} i_{4}}=L_{1} \cap L_{1 i_{2} i_{3} i_{4}}$. Then the set $\left\{P_{i_{2} i_{3} i_{4}}\right\}$ is invariant under the group $\bar{G}_{1} \cong \mathrm{A}_{5}$ and consists of at most $\left(\begin{array}{l}5 \\ 3\end{array}\right)=10$ points. This is impossible by Lemma 2.9 ,

Finally, suppose that for some 5 of the lines of $\mathrm{L}$ (say, for $L_{2}, \ldots, L_{6}$ ) there exists a line $L$ that intersects all 5 of them. The above argument implies that there are at most 2 lines intersecting $L_{2}, \ldots, L_{6}$, so that $L$ is invariant under the group $\bar{G}_{1} \cong \mathrm{A}_{5}$. Since the lines of $\mathrm{L} \cup \mathrm{L}^{\prime}$ are pairwise disjoint, the line $L$ coincides with neither $L_{1}$ nor $L_{1}^{\prime}$. Hence there are at least 3 lines in $\mathbb{P}^{3}$ that are invariant under $\bar{G}_{1}$, which contradicts Remark 3.5.

The same arguments apply if one replaces the lines of $\mathrm{L}$ by the lines of $\mathrm{L}^{\prime}$.

Recall that several lines in $\mathbb{P}^{3}$ are said to lie in a linear complex if the corresponding points of the Grassmaniann $\operatorname{Gr}(2,4) \subset \mathbb{P}^{5}$ lie in a hyperplane section.

Lemma 4.2. Neither $L_{1}, L_{2}, L_{3}, L_{4}, L_{5}, L_{6}$ nor $L_{1}^{\prime}, L_{2}^{\prime}, L_{3}^{\prime}, L_{4}^{\prime}, L_{5}^{\prime}, L_{6}^{\prime}$ lie in a linear complex.

Proof. It follows from Remark 3.4 that the space $\Lambda^{2}(V) \cong \mathbb{C}^{6}$ is the permutation representation of the group $\bar{G} \cong \mathrm{A}_{6}$. The natural action of the group $\bar{G}$ on $\operatorname{Gr}(2,4) \subset \mathbb{P}^{5} \cong \mathbb{P}\left(\Lambda^{2}(V)\right)$ arises from this representation. Let us identify $\bar{G}$ with a subgroup in $\operatorname{Aut}\left(\mathbb{P}^{5}\right)$. Then there is a unique $\bar{G}$-invariant hyperplane $H \subset \mathbb{P}^{5}$. We may assume that $\operatorname{Gr}(2,4) \cap H$ is given by

$$
\sum_{i=0}^{5} x_{i}=\sum_{i=0}^{5} x_{i}^{2}=0 \subset \mathbb{P}^{5} \cong \operatorname{Proj}\left(\mathbb{C}\left[x_{0}, x_{1}, x_{2}, x_{3}, x_{4}, x_{5}\right]\right),
$$

and $H$ is given by $\sum_{i=0}^{5} x_{i}=0$. Let $P \in \operatorname{Gr}(2,4)$ be a point that corresponds to the line $L_{1} \subset \mathbb{P}^{3}$, and let $\bar{G}_{1}$ be the stabilizer subgroup in $\bar{G}$ of the point $P$. Then $\bar{G}_{1} \cong \mathrm{A}_{5}$, and the embedding $\bar{G}_{1} \subset \bar{G}$ is standard. If the $\bar{G}$-orbit of the point $P$ is contained in a hyperplane, then this hyperplane must be $H$, which implies that the $\bar{G}$-orbit of the point $P$ must contain a point in $\mathbb{P}^{5}$ that is given by $x_{0}=\ldots=x_{4}=-x_{5} / 5$, which is impossible, because this point does not belong to the intersection $\operatorname{Gr}(2,4) \cap H$.

Now we are ready to formulate the main technical result of this section.

Theorem 4.3. Let $\mathcal{M}$ be a (non-empty) $\bar{G}$-invariant linear system on $\mathbb{P}^{3}$ that does not have fixed components, and let $\lambda$ be a positive rational number such that $\lambda \mathcal{M} \sim \mathbb{Q}-K_{\mathbb{P}^{3}}$. Suppose that $\left(\mathbb{P}^{3}, \lambda \mathcal{M}\right)$ is canonical at a general point of every line $L_{1}, \ldots, L_{6}, L_{1}^{\prime}, \ldots, L_{6}^{\prime}$. Then $\left(\mathbb{P}^{3}, \lambda \mathcal{M}\right)$ is canonical.

Before proving Theorem 4.3 , let us show that it implies that $\mathbb{P}^{3}$ is $\bar{G}$-birationally rigid and $\operatorname{Bir}^{\bar{G}}\left(\mathbb{P}^{3}\right)$ is isomorphic to a free product of $\mathrm{S}_{6}$ and $\mathrm{S}_{6}$ with an amalgamated subgroup $\mathrm{A}_{6}$. To do this, will use the lines $L_{1}, \ldots, L_{6}, L_{1}^{\prime}, \ldots, L_{6}^{\prime}$ to prove that $A u{ }^{\bar{G}}\left(\mathbb{P}^{3}\right)=\hat{G}$ and to construct two $\bar{G}$-equivariant birational non-biregular involutions of $\mathbb{P}^{3}$ that was described in [45]. The proof of $\bar{G}$-birational rigidity of $\mathbb{P}^{3}$ and description of the group $\operatorname{Bir}^{\bar{G}}\left(\mathbb{P}^{3}\right)$ crucially depend on these birational involutions.

Lemma 4.4. The equality $\operatorname{Aut}^{\bar{G}}\left(\mathbb{P}^{3}\right)=\hat{G}$ holds.

Proof. Put $\tilde{G}=\operatorname{Aut}^{\bar{G}}\left(\mathbb{P}^{3}\right)$. Then the curve $\sum_{i=1}^{6} L_{i}+\sum_{i=1}^{6} L_{i}^{\prime}$ is $\tilde{G}$-invariant, and the group $\bar{G}$ is a normal subgroup of the group $\tilde{G}$. There is a subgroup $\breve{G} \subset \tilde{G}$ of index 2 such that $\sum_{i=1}^{6} L_{i}$ and $\sum_{i=1}^{6} L_{i}^{\prime}$ are $\breve{G}$-invariant.

Let $Y$ be the Hierholzer surface of the lines $L_{1}, L_{2}, L_{3}, L_{4}, L_{5}, L_{6}$ (see [46, Section 2.1]). Then the surface $Y$ is $\breve{G}$-invariant and there exists a monomorphism $\xi: \breve{G} \rightarrow \operatorname{Aut}(Y)$. Moreover, the surface $Y$ is birational to a surface of general type (see [46, Theorem 2.1]), which implies 
that $\breve{G}$ is a finite group (see e.g.[21, Theorem 1.1]). Thus $\tilde{G}$ is a finite group. Hence, we have $\operatorname{lct}\left(\mathbb{P}^{3}, \tilde{G}\right) \geqslant \operatorname{lct}\left(\mathbb{P}^{3}, \bar{G}\right) \geqslant 5 / 4$ by [10, Theorem 4.13$]$.

Let $\phi: \mathrm{SL}_{4}(\mathbb{C}) \rightarrow \operatorname{Aut}\left(\mathbb{P}^{3}\right)$ be the natural projection. Then there is a finite subgroup $G \subset$ $\mathrm{SL}_{4}(\mathbb{C})$ such that $\tilde{G}=\phi(G)$. Note that $G$ may not be uniquely defined. However, by [10, Theorem 4.13], we may assume that $G$ is one of the eight groups described in [10, Lemma 4.12]. Suppose that $\hat{G} \subsetneq \tilde{G}$. Then $G \neq 2 . \mathrm{S}_{6}$.

Recall that $\bar{G}$ is a (non-trivial) normal subgroup in $\tilde{G}$. Hence $G$ cannot be isomorphic to any of the groups $2 . \mathrm{A}_{7}$ and $\mathrm{PSp}_{4}\left(\mathbb{F}_{3}\right)$, because the images of these groups in $\mathrm{PGL}_{4}(\mathbb{C})$ are simple groups. Moreover, $G \neq 2 . \mathrm{A}_{6}$, since there is a subgroup $\hat{G} \subset \tilde{G}$ isomorphic to $\mathrm{S}_{6}$.

For each of the four remaining groups from [10, Lemma 4.12] one has (see [36]) an exact sequence of groups

$$
1 \longrightarrow \mathbb{Z}_{2}^{4} \stackrel{\alpha}{\longrightarrow} \tilde{G} \stackrel{\beta}{\longrightarrow} \Gamma,
$$

where $\Gamma$ is a subgroup of the group $\mathrm{S}_{6}$. Thus $\Gamma \cong \mathrm{S}_{6}$ because $\tilde{G} \supset \hat{G} \cong \mathrm{S}_{6}$. Let $e$ be the identity element in $\tilde{G}$. Then $\bar{G} \cap \operatorname{im}(\alpha)=e$, because $\bar{G}$ is a simple group, and $\bar{G}$ is a normal subgroup of the group $\tilde{G}$. Hence the subgroup of $\tilde{G}$ generated by $\bar{G} \cong \mathrm{A}_{6}$ and $\operatorname{im}(\alpha) \cong \mathbb{Z}_{2}^{4}$ is a direct product of the latter groups. This leads to a contradiction because the action of $\Gamma$ on $\mathbb{Z}_{2}^{4}$ is faithful (see [36]).

Let $H$ be a general hyperplane in $\mathbb{P}^{3}$.

Lemma 4.5. There is a $\bar{G}$-equivariant birational non-biregular involution $\iota \in \operatorname{Bir}\left(\mathbb{P}^{3}\right)$ such that $\iota(H)$ is a surface of degree 19 that has singularity of multiplicity 5 in a general point of every line $L_{1}, \ldots, L_{6}$, and the group generated by $\bar{G}$ and $\iota$ is isomorphic to $\mathrm{S}_{6}$.

Proof. Let $\mathcal{H}$ be a linear subsystem of the linear system $\left|\mathcal{O}_{\mathbb{P} 3}(4)\right|$ consisting of surfaces that pass through the lines $L_{1}, L_{2}, L_{3}, L_{4}, L_{5}, L_{6}$. Then it follows from [45] and [46. Theorem 2.4] that $\mathcal{H}$ does not have fixed components, and $\mathcal{H}$ gives a rational map $\psi: \mathbb{P}^{3} \rightarrow V$, where $V$ is a quartic threefold in $\mathbb{P}^{4}$. Let $\alpha: U \rightarrow \mathbb{P}^{3}$ be a blow up along $L_{1}, L_{2}, L_{3}, L_{4}, L_{5}, L_{6}$. Then there is a commutative diagram

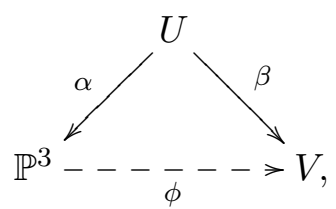

where $\beta$ is a birational morphism that contracts finitely many curves. It follows from 46, Theorem 2.4] that $V \subset \mathbb{P}^{4}$ can be given by the equation 4

$$
\begin{gathered}
5\left(y w-z t+x y+x w-x z-x t-x^{2}\right)^{2}-20 x y w(w-x+y-z-t) \\
\| \\
\left(3 x^{2}+2 y^{2}+2 z^{2}+2 t^{2}+2 w^{2}-3 x y+3 x z+3 x t-3 x w-2 y z-2 y t+y w+z t-2 z w-2 t w\right)^{2}
\end{gathered}
$$

in appropriate homogeneous coordinates $[x: y: z: t: w]$ in $\mathbb{P}^{4}$. By [45] we know that the singular locus of the threefold $V$ consists of 36 nodes, and the morphism $\beta$ contracts the proper transforms of 30 lines in $\mathbb{P}^{3}$ each of whom intersects 4 lines among $L_{1}, L_{2}, L_{3}, L_{4}, L_{5}, L_{6}$, and proper transforms of 6 twisted cubics in $\mathbb{P}^{3}$ each of whom has the lines $L_{1}, L_{2}, L_{3}, L_{4}, L_{5}, L_{6}$ as chords.

The map $\phi$ is $\bar{G}$-equivariant. We can identify $\bar{G}$ with a subgroup in $\operatorname{Aut}(V)$ and with a subgroup in $\operatorname{Aut}\left(\mathbb{P}^{4}\right)$. By Lemma [3.7, there are no $\bar{G}$-fixed points in $\mathbb{P}^{4}$. So, the action of

\footnotetext{
${ }^{4}$ Note that the quartic threefold $V \subset \mathbb{P}^{4}$ is determinantal (see [45], 37, Example 6.4.2]).
} 
the group $\bar{G}$ on $\mathbb{P}^{4}$ arises from its irreducible five-dimensional representation (cf. Remark 3.4). Hence, there is a subgroup $\bar{\Gamma} \subset \operatorname{Aut}(V)$ such that $\bar{G} \subset \bar{\Gamma} \cong \mathrm{S}_{6}$. Let $\theta$ be an involution in $\bar{\Gamma}$ such that $\theta \notin \bar{G}$. Put $\iota=\phi^{-1} \circ \theta \circ \phi \in \operatorname{Bir}^{\bar{G}}\left(\mathbb{P}^{3}\right)$. Then it follows from the construction of $\iota$ that $\langle\iota, \bar{G}\rangle \cong \mathrm{S}_{6}$.

Let us show that $\iota$ is not biregular. Suppose that this is not true. Let us identify the subgroup $\langle\iota, \bar{G}\rangle$ with $\bar{\Gamma} \cong \mathrm{S}_{6}$. Then $\sum_{i=1}^{6} L_{i}$ must be $\bar{\Gamma}$-invariant. Let $\bar{G}_{1} \subset \bar{G}$ and $\bar{\Gamma}_{1} \subset \bar{\Gamma}$ be stabilizers of the line $L_{1}$. Then $A_{5} \cong \bar{G}_{1} \subset \bar{\Gamma}_{1} \cong \mathrm{S}_{5}$, and there are natural homomorphisms $\zeta: \bar{G}_{1} \rightarrow \operatorname{Aut}\left(L_{1}\right)$ and $\eta: \bar{\Gamma}_{1} \rightarrow \operatorname{Aut}\left(L_{1}\right)$. Thus, we have $\mathrm{A}_{5} \cong \operatorname{im}(\zeta) \subseteq \operatorname{im}(\eta)$, which gives $\operatorname{im}(\eta) \cong \mathrm{S}_{5}$. But $\operatorname{Aut}\left(L_{1}\right) \cong \mathrm{PGL}_{2}(\mathbb{C})$ contains no subgroups isomorphic to $\mathrm{S}_{5}$. The obtained contradiction shows that $\iota$ is not biregular.

Put $\tau=\beta^{-1} \circ \theta \circ \beta \in \operatorname{Bir}(U)$. Then $\tau$ is a composition of flops, and the commutative diagram

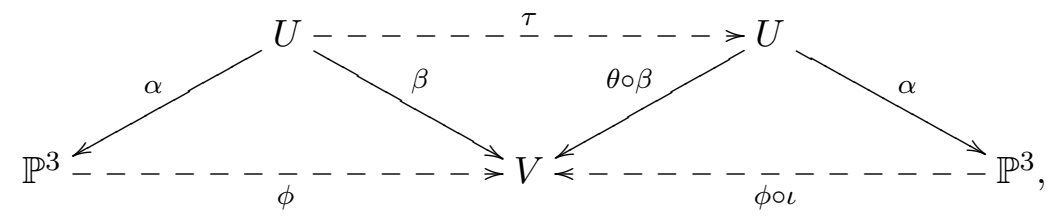

is a $\bar{G}$-equivariant Sarkisov link of type II (see [13, Definition 3.4]). The involution $\tau$ is biregular outside of the curves contracted by $\beta$. Then $\tau$ naturally acts on the group $\operatorname{Pic}(U)$. Moreover, this action is nontrivial, because $\iota$ is not biregular.

Put $\hat{H}=\alpha^{*}(H)$. Let $E_{k}$ be the $\alpha$-exceptional divisor such that $\alpha\left(E_{k}\right)=L_{k}$. Then

$$
\tau^{*}(\hat{H}) \cdot \tau^{*}(\hat{H}) \cdot\left(4 \hat{H}-\sum_{i=1}^{6} E_{i}\right)=4,
$$

because $\tau^{*}\left(K_{U}\right) \sim K_{U}$ and $\beta$ contracts finitely many curves. Similarly, we see that

$$
\tau^{*}(\hat{H}) \cdot \tau^{*}\left(E_{k}\right) \cdot\left(4 \hat{H}-\sum_{i=1}^{6} E_{i}\right)=1, \tau^{*}\left(E_{i}\right) \cdot \tau^{*}\left(E_{k}\right) \cdot\left(4 \hat{H}-\sum_{i=1}^{6} E_{i}\right)=\left\{\begin{array}{l}
-2 \text { if } i=k, \\
0 \text { if } i \neq k,
\end{array}\right.
$$

which immediately implies that either $\tau^{*}(\hat{H}) \sim \hat{H}$ and $\tau^{*}\left(E_{k}\right)=E_{k}$, or

$$
\left\{\begin{array}{l}
\tau^{*}(\hat{H}) \sim 19 \hat{H}-5 \sum_{i=1}^{6} E_{i}, \\
\tau^{*}\left(E_{k}\right) \sim 12 \hat{H}-4 E_{k}-\sum_{i \neq k} 3 E_{i},
\end{array}\right.
$$

Since $\iota$ is not biregular, the involution $\tau$ must act non-trivially on $\operatorname{Pic}(U)$. Thus, we see that (4.6) holds, which implies, in particular, that $\iota(H)$ is a surface of degree 19 that has singularity of multiplicity 5 in a general point of every line $L_{1}, \ldots, L_{6}$, because $\iota(H)=\alpha \circ \tau(\hat{H})$.

Put $\iota^{\prime}=\nu \circ \iota \circ \nu^{-1}$ for any $\nu \in \hat{G} \cong \mathrm{S}_{6}$ such that $\nu \notin \bar{G}$. Then $\iota^{\prime}$ is a $\bar{G}$-equivariant birational non-biregular involution of $\mathbb{P}^{3}$ such that $\iota^{\prime}(T)$ is a surface of degree 19 that has singularity of multiplicity 5 in a general point of every line $L_{1}^{\prime}, \ldots, L_{6}^{\prime}$, and the group generated by $\bar{G}$ and $\iota^{\prime}$ is also isomorphic to $\mathrm{S}_{6}$. Note that the choice of $\iota$ and $\iota^{\prime}$ is not unique. Put $\Gamma=\left\langle\iota, \iota^{\prime}, \hat{G}\right\rangle$. Let us use birational involutions $\iota$ and $\iota^{\prime}$ together with Theorem 4.3 to prove

Lemma 4.7. Let $\mathcal{M}$ be a (non-empty) $\bar{G}$-invariant linear system on $\mathbb{P}^{3}$ that does not have fixed components. Then there are $\rho \in \Gamma$ and $\mu \in \mathbb{Q}_{>0}$ such that $\mu \rho(\mathcal{M}) \sim_{\mathbb{Q}}-K_{\mathbb{P}^{3}}$, and $\left(\mathbb{P}^{3}, \mu \rho(\mathcal{M})\right)$ is canonical. 
Proof. Take $\lambda \in \mathbb{Q}_{>0}$ such that $\lambda \mathcal{M} \sim \mathbb{Q}-K_{\mathbb{P}^{3}}$. If $\left(\mathbb{P}^{3}, \lambda \mathcal{M}\right)$ is canonical, then we are done. Thus, we may assume that $\left(\mathbb{P}^{3}, \lambda \mathcal{M}\right)$ is not canonical. By Theorem 4.3 , the log pair $\left(\mathbb{P}^{3}, \lambda \mathcal{M}\right)$ is not canonical at a general point of one line among $L_{1}, \ldots, L_{6}, L_{1}^{\prime}, \ldots, L_{6}^{\prime}$.

Without loss of generality, we may assume that $\left(\mathbb{P}^{3}, \lambda \mathcal{M}\right)$ is not canonical at a general point of the line $L_{1}$. Then $\left(\mathbb{P}^{3}, \lambda \mathcal{M}\right)$ is not canonical at a general point of the lines $L_{2}, \ldots, L_{6}$, since $\mathcal{M}$ is $\bar{G}$-invariant. Let $M$ be a general surface in $\mathcal{M}$. Then $\operatorname{mult}_{L_{i}}(M)>1 / \lambda$ for every $i \in\{1, \ldots, 6\}$ (see [14, Exercise 6.18]). Note that $M$ is a surface of degree $d=4 / \lambda$. Let $\tilde{d}$ be the degree of the surface $\iota(M)$. Put $\tilde{\lambda}=4 / \tilde{d}$. Then $\tilde{\lambda} \iota(\mathcal{M}) \sim_{\mathbb{Q}}-K_{\mathbb{P} 3}$. Moreover, it follows from Lemma 4.5 that

$$
\tilde{d}=19 d-12 \sum_{i=1}^{6} \operatorname{mult}_{L_{i}}(M)<19 d-18 d=d .
$$

If $\left(\mathbb{P}^{3}, \tilde{\lambda} \iota(\mathcal{M})\right)$ is canonical, then we are done. If it is not canonical, then it follows from Theorem 4.3 that $\left(\mathbb{P}^{3}, \tilde{\lambda} \iota(\mathcal{M})\right)$ is not canonical at a general point of one line among $L_{1}, \ldots, L_{6}, L_{1}^{\prime}, \ldots, L_{6}^{\prime}$. Thus, we can iterate the above process. Since $\tilde{d}<d$, our iterations must terminate in at most $d-1$ steps, which completes the proof.

Now we ready to prove

Theorem 4.8. The variety $\mathbb{P}^{3}$ is $\bar{G}$-birationally rigid and $\operatorname{Bir}^{\bar{G}}\left(\mathbb{P}^{3}\right)=\Gamma$.

Proof. Suppose that there exists a rational normal threefold $V$ with at most terminal singularities that admits a faithful action of the group $\bar{G}$ such that there are a $\bar{G}$-equivariant Mori fibration $\pi: V \rightarrow S$ and a $\bar{G}$-equivariant birational map $\xi: V \rightarrow \mathbb{P}^{3}$. Let $D$ be a sufficiently general very ample divisor on $V$. Put $\mathcal{M}=\xi(|D|)$. Then $\mathcal{M}$ does not have fixed components and is $\bar{G}$-invariant. Thus, it follows from Lemma 4.7 that there are $\rho \in \Gamma$ and $\mu \in \mathbb{Q}_{>0}$ such that $\mu \rho(\mathcal{M}) \sim_{\mathbb{Q}}-K_{\mathbb{P}^{3}}$, and $\left(\mathbb{P}^{3}, \mu \rho(\mathcal{M})\right)$ is canonical. Then $\rho \circ \xi$ is biregular by [12, Theorem 4.2]. In particular, we see that $V \cong \mathbb{P}^{3}$ and $S$ is a point. Since all subgroups in $\operatorname{Aut}\left(\mathbb{P}^{3}\right)$ that are isomorphic to $\bar{G}$ are conjugate to each other, we see that $\mathbb{P}^{3}$ is $\bar{G}$-birationally rigid and $\operatorname{Bir}^{\bar{G}}\left(\mathbb{P}^{3}\right)=\Gamma$.

Before proving Theorem 4.3, let us use it one more time to prove

Theorem 4.9. The group $\Gamma$ is the free product of $\hat{G}$ and $\langle\iota, \bar{G}\rangle$ with amalgamated subgroup $\bar{G}$.

Proof. Let $\xi$ be a birational automorphism in $\operatorname{Bir}^{\bar{G}}\left(\mathbb{P}^{3}\right)$ such that $\xi \notin \hat{G}$. Put $\mathcal{M}=\xi(|T|)$ and take $\lambda \in \mathbb{Q}$ such that $\lambda \mathcal{M} \sim_{\mathbb{Q}}-K_{\mathbb{P}^{3}}$. Then $\left(\mathbb{P}^{3}, \lambda \mathcal{M}\right)$ is not canonical by [12, Theorem 4.2]. By Theorem 4.3, the log pair $\left(\mathbb{P}^{3}, \lambda \mathcal{M}\right)$ is not canonical at a general point of one line among $L_{1}, \ldots, L_{6}, L_{1}^{\prime}, \ldots, L_{6}^{\prime}$. Arguing as in the proof of Lemma 4.7, we see that there exists a combination

$$
\zeta=\underbrace{\ldots \circ \iota \circ \iota^{\prime} \circ \iota \circ \iota^{\prime} \circ \iota \circ \ldots}_{m \text { times }}
$$

such that $\left(\mathbb{P}^{3}, \mu \zeta(\mathcal{M})\right)$ is canonical, where $\mu$ is a positive rational number such that one has $\mu \zeta(\mathcal{M}) \sim_{\mathbb{Q}}-K_{\mathbb{P}^{3}}$. Then $\zeta \circ \xi$ is biregular by [12, Theorem 4.2], which implies that $\zeta \circ \xi \in \hat{G}$ by Lemma 4.4 .

Let us show that $\zeta$ is uniquely determined by $\xi$ and the algorithm hidden in the proof Lemma 4.7 ,

Let $M$ be a general surface in $\mathcal{M}$, and let $d$ be its degree. Then $d=4 / \lambda$. Arguing as in the proof of Lemma 4.7, we see that if $\left(\mathbb{P}^{3}, \lambda \mathcal{M}\right)$ is not canonical at a general point of one line among $L_{1}, \ldots, L_{6}$, then the degree of $\iota(M)$ is smaller than $d$. Similarly, we see that if $\left(\mathbb{P}^{3}, \lambda \mathcal{M}\right)$ is not canonical at a general point of one line among $L_{1}^{\prime}, \ldots, L_{6}^{\prime}$, then the degree of $\iota^{\prime}(M)$ is smaller than $d$. We can use this to construct $\xi$ step by step. Thus, to show that $\zeta$ is uniquely 
determined by $\xi$, we must show that $\left(\mathbb{P}^{3}, \lambda \mathcal{M}\right)$ can not be non-canonical at a general point of one line among $L_{1}, \ldots, L_{6}$ and the same time at a general point of one line among $L_{1}^{\prime}, \ldots, L_{6}^{\prime}$.

Suppose that $\left(\mathbb{P}^{3}, \lambda \mathcal{M}\right)$ is not canonical at a general point of one line among $L_{1}, \ldots, L_{6}$ and the same time at a general point of one line among $L_{1}^{\prime}, \ldots, L_{6}^{\prime}$. Then $\left(\mathbb{P}^{3}, \lambda \mathcal{M}\right)$ is not canonical at a general point of every line among $L_{1}, \ldots, L_{6}, L_{1}^{\prime}, \ldots, L_{6}^{\prime}$, since $\mathcal{M}$ is $\bar{G}$-invariant. Then mult $_{L_{i}}(M)>1 / \lambda$ and $\operatorname{mult}_{L_{i}^{\prime}}(M)>1 / \lambda$ for every $i \in\{1,2, \ldots, 6\}$. Let $\Pi$ be a general plane in $\mathbb{P}^{3}$ that contains the line $L_{1}$. Put $\left.\mathcal{M}\right|_{\Pi}=\operatorname{mult}_{L_{1}}(M) L_{1}+\mathcal{B}$, where $\mathcal{B}$ is a linear system on $\Pi$ that does not have fixed components. Let $B_{1}$ and $B_{2}$ be general curves in $\mathcal{B}$, let $O_{i}$ be the point $\Pi \cap L_{i}$ for every $i \in\{2, \ldots, 6\}$, and let $O_{i}^{\prime}$ be the point $\Pi \cap L_{i}^{\prime}$ for every $i \in\{1, \ldots, 6\}$. Then $\operatorname{mult}_{O_{i}}\left(B_{1}\right)=\operatorname{mult}_{O_{i}}\left(B_{2}\right)=\operatorname{mult}_{L_{1}}(M)$ for every $i \in\{2, \ldots, 6\}$, and mult $_{O_{i}^{\prime}}\left(B_{1}\right)=$ mult $_{O_{i}^{\prime}}\left(B_{2}\right)=$ mult $_{L_{1}^{\prime}}(M)$ for every $i \in\{1, \ldots, 6\}$. Then

$$
B_{1} \cdot B_{2} \geqslant \sum_{i=2}^{6} \operatorname{mult}_{O_{i}}\left(B_{1}\right) \text { mult }_{O_{i}}\left(B_{2}\right)+\sum_{i=1}^{6} \text { mult }_{O_{i}^{\prime}}\left(B_{1}\right) \text { mult }_{O_{i}^{\prime}}\left(B_{2}\right)>\frac{11}{\lambda^{2}},
$$

because mult $_{L_{1}}(M)>1 / \lambda$ and mult $_{L_{1}^{\prime}}(M)>1 / \lambda$. On the other hand, we have

$$
B_{1} \cdot B_{2}=\left(\left.\frac{4}{\lambda} \Pi\right|_{\Pi}-\operatorname{mult}_{L_{1}}(M) L_{1}\right)^{2}=\left(\frac{4}{\lambda}-\operatorname{mult}_{L_{1}}(M)\right)^{2}<\frac{9}{\lambda^{2}}
$$

which is a contradiction. Thus we see that $\left(\mathbb{P}^{3}, \lambda \mathcal{M}\right)$ is not canonical either at a general point of every line among $L_{1}, \ldots, L_{6}$, or at a general point of every line among $L_{1}^{\prime}, \ldots, L_{6}^{\prime}$. But it can not be non-canonical at a general point of all these 12 lines.

Recall that we defined $\iota^{\prime}$ ad $\nu \circ \iota \circ \nu^{-1}$ for some $\nu \in \hat{G}$ such that $\nu \notin \bar{G}$. Without loss of generality we may assume that $\nu$ is an involution. Thus, we see that every $\alpha \in \operatorname{Bir}^{\bar{G}}\left(\mathbb{P}^{3}\right)$ can be uniquely written as

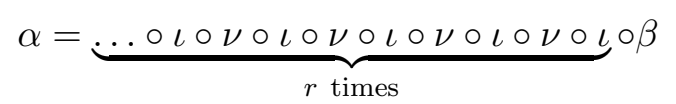

for some $\beta \in \hat{G}$ such that $r=0$ if and only if $\alpha \in \hat{G}$. The latter implies that $\Gamma$ is the free product of the groups $\hat{G}=\langle\nu, \bar{G}\rangle \cong \mathrm{S}_{6}$ and $\langle\iota, \bar{G}\rangle \cong \mathrm{S}_{6}$ with amalgamated subgroup $\bar{G} \cong \mathrm{A}_{6}$.

Remark 4.10. Note that $\hat{G} \not \subset\left\langle\iota, \iota^{\prime}, \bar{G}\right\rangle$ (see the proof of Theorem 4.9).

In the rest of this section, we prove Theorem 4.3. Let $\mathcal{M}$ be a (non-empty) $\bar{G}$-invariant linear system on $\mathbb{P}^{3}$ that does not have fixed components, and let $\lambda$ be a positive rational number such that $\lambda \mathcal{M} \sim \mathbb{Q}-K_{\mathbb{P}^{3}}$. Suppose that $\left(\mathbb{P}^{3}, \lambda \mathcal{M}\right)$ is canonical at a general point of every line $L_{1}, \ldots, L_{6}, L_{1}^{\prime}, \ldots, L_{6}^{\prime}$. We must prove that $\left(\mathbb{P}^{3}, \lambda \mathcal{M}\right)$ is canonical.

Suppose that the log pair $\left(\mathbb{P}^{3}, \lambda \mathcal{M}\right)$ is not canonical. Let us seek for a contradiction. By Corollary 2.3 there is $\mu \in \mathbb{Q}$ such that $\mu<2 \lambda$ and $\left(\mathbb{P}^{3}, \mu \mathcal{M}\right)$ is strictly log canonical. Let $S \subset X$ be a minimal center in $\mathbb{L} \mathbb{C} S\left(\mathbb{P}^{3}, \mu \mathcal{M}\right)$. Then $S$ is not a surface, since $\mathcal{M}$ has no fixed components.

Recall that we denote by $H$ a general hyperplane in $\mathbb{P}^{3}$. By Lemma 2.13, there is $\delta \in \mathbb{Q}$ and there is a $\bar{G}$-invariant effective $\mathbb{Q}$-divisor $D \sim_{\mathbb{Q}} \delta H$ on the threefold $\mathbb{P}^{3}$ such that $0<\delta<8$, the log pair $\left(\mathbb{P}^{3}, D\right)$ is log canonical and the set $\mathbb{L} \mathbb{C} \mathbb{S}\left(\mathbb{P}^{3}, D\right)$ consists of all irreducible components of the $\bar{G}$-orbit of $S$.

Remark 4.11. It follows from the proof of Lemma 2.13 that we may assume that $\operatorname{mult}_{L_{i}}(D)<2$ and mult $_{L_{i}^{\prime}}(D)<2$ for every $i \in\{1,2, \ldots, 6\}$, since $\left(\mathbb{P}^{3}, \lambda \mathcal{M}\right)$ is canonical at a general point of every line $L_{1}, \ldots, L_{6}, L_{1}^{\prime}, \ldots, L_{6}^{\prime}$.

Let $\mathcal{I}$ be the multiplier ideal sheaf of the $\log$ pair $\left(\mathbb{P}^{3}, D\right)$, and let $\mathcal{L}$ be the log canonical singularities subscheme of the log pair $\left(\mathbb{P}^{3}, D\right)$. Then it follows from Theorem 2.4 that

$$
h^{0}\left(\mathcal{O}_{\mathcal{L}} \otimes \mathcal{O}_{\mathbb{P}^{3}}(4 H)\right)=h^{0}\left(\mathcal{O}_{\mathbb{P}^{3}}(4 H)\right)-h^{0}\left(\mathcal{O}_{\mathbb{P}^{3}}(4 H) \otimes \mathcal{I}\right)=35-h^{0}\left(\mathcal{O}_{\mathbb{P}^{3}}(4 H) \otimes \mathcal{I}\right)
$$


Lemma 4.13. The center $S$ is a curve.

Proof. If $S$ is a point, then $\left|\operatorname{LCS}\left(\mathbb{P}^{3}, D\right)\right| \leqslant 35$ by (4.12), which contradicts Lemma 3.8 .

By Theorem 2.8, the curve $S$ is a smooth curve in $\mathbb{P}^{3}$ of degree $d$ and genus $g$ such that

$$
g \leqslant 2 d
$$

Put $q=h^{0}\left(\mathcal{O}_{\mathbb{P}^{3}}(4 H) \otimes \mathcal{I}\right)$. Let $Z$ be the $\bar{G}$-orbit of the curve $S$, let $r$ be the number of irreducible components of $Z$. Then $Z=\mathcal{L}$, since $\left(\mathbb{P}^{3}, D\right)$ is $\log$ canonical. Moreover, the curve $Z$ is smooth by Lemma 2.7 , and $Z$ is not contained in a hyperplane in $\mathbb{P}^{3}$ by Remark 3.4 .

Lemma 4.15. The equality $r(4 d-g+1)=35-q$ holds.

Proof. The equality follows from (4.12) and the Riemann-Roch theorem, because $2 d \geqslant g$.

Corollary 4.16. The inequality $g \leqslant 34$ holds.

Lemma 4.17. Suppose that $1 \leqslant q \leqslant 7$. Then $q \in\{5,6\}$.

Proof. Put $W_{4}=H^{0}\left(\mathcal{O}_{\mathbb{P}^{3}}(4 H) \otimes \mathcal{I}\right)$. Since the center of $G$ acts trivially on polynomials of even degree, the space $W_{4}$ has a natural structure of a $\bar{G}$-representation. Suppose that $q \notin\{5,6\}$. Then $W_{4}$ has a trivial subrepresentation of the group $\bar{G}$ by dimension reasons (see [15]), which is impossible by Lemma 3.7

Lemma 4.18. Suppose that $r \neq 1$. Then $r=6$ and $d=1$.

Proof. Since $r \leqslant 35$ by Lemma 4.15, one has $r \in\{6,10,15,20,30\}$ by Lemma 3.3, In particular, the inequality $q \geqslant 1$ holds by Lemma 4.15 .

Suppose that $q \geqslant 6$. Then $r(4 d-g+1)<30$ by Lemma 4.15. We see that $g+1 \leqslant 4 d-g+1<5$, which implies that $g \leqslant 3$. Then $d=1$ and $g=0$, so that $4 d-g+1=5$, which is a contradiction.

By Lemma 4.17 we may assume that $q=5$. Then $r \in\{6,10,15,30\}$ by Lemma 4.15.

If $r=30$, then $g+1 \leqslant 4 d-g+1=1$ by Lemma 4.15, which is a contradiction.

If $r=15$, then $g+1 \leqslant 4 d-g+1=2$ by Lemma 4.15, which leads to a contradiction.

If $r=10$, then $g+1 \leqslant 4 d-g+1=3$ by Lemma 4.15, which leads to a contradiction.

If $r=6$, then $g+1 \leqslant 4 d-g+1=5$ by Lemma 4.15, which gives $g=0$ and $d=1$.

Lemma 4.19. The equality $r=1$ holds.

Proof. Suppose that $r \neq 1$. Then $Z$ is a disjoint union of 6 lines by Lemma 4.18, which implies that either $Z=L_{1}+L_{2}+L_{3}+L_{4}+L_{5}+L_{6}$, or $Z=L_{1}^{\prime}+L_{2}^{\prime}+L_{3}^{\prime}+L_{4}^{\prime}+L_{5}^{\prime}+L_{6}^{\prime}$.

Without loss of generality, we may assume that $S=L_{1}$ and $Z=L_{1}+L_{2}+L_{3}+L_{4}+L_{5}+L_{6}$.

Let $\bar{F} \subset \bar{G}$ be the stabilizer of $L_{1}$. Then $\bar{F} \cong \mathrm{A}_{5}$. Let $\pi: U \rightarrow \mathbb{P}^{3}$ be the blow up of $L_{1}$. Then

$$
K_{U}+\bar{D}+\left(\operatorname{mult}_{L_{1}}(D)-1\right) E \sim_{\mathbb{Q}} \pi^{*}\left(K_{\mathbb{P}^{3}}+D\right),
$$

where $E$ is the $\pi$-exceptional divisor, and $\bar{D}$ is the proper transform of $D$ on $U$. One has $2>\operatorname{mult}_{L_{1}}(D)>1$ by Remark 4.11. The group $\bar{F}$ naturally acts on $E$ so that the divisor $\left.\bar{D}\right|_{E}$ is $\bar{F}$-invariant.

We can identify the surface $E$ with a smooth quadric in $\mathbb{P}^{3}$. The action of the group $\bar{F}$ extends to the ambient space $\mathbb{P}^{3}$. Note that this action arises from the standard four-dimensional representation of the group $\bar{F} \cong \mathrm{A}_{5}$.

It follows from the inequality $\operatorname{mult}_{L_{1}}(D)<2$ that the set $\mathbb{L} \mathbb{C S}\left(U, \bar{D}+\left(\operatorname{mult}_{L_{1}}(D)-1\right) E\right)$ contains an irreducible reduced curve $C \subset E$ such that $\pi(C)=L_{1}$. One has $C \in \mathbb{L} \mathbb{C}\left(E,\left.\bar{D}\right|_{E}\right)$ by Theorem 2.6. which is impossible by Lemma 2.17.

We see that $r=1$, so that $Z=S$. By Lemma 2.10 one has $g \in\{31,25,19,16,10\}$.

Lemma 4.20. The inequality $g \neq 31$ holds. 
Proof. Suppose that $g=31$. Then it follows from Lemma 4.15 that

$$
d=\frac{65-q}{4},
$$

which gives $q>0$. Hence $q \geqslant 5$ by Lemma 4.17 and thus $d \leqslant 15$ by (4.21). By (4.14) we have $g / 2 \leqslant d \leqslant 15$, which is impossible.

Lemma 4.22. The inequality $g \neq 25$ holds.

Proof. Suppose that $g=25$. Then it follows from Lemma 4.15 that

$$
d=\frac{59-q}{4},
$$

which gives $q>0$. Hence $q \geqslant 11$ by Lemma 4.17 and thus $d \leqslant 15$ by (4.23). By (4.14) we have $g / 2 \leqslant d \leqslant 12$, which is impossible.

Lemma 4.24. The inequality $g \neq 19$ holds.

Proof. Suppose that $g=19$. Then $S$ is not contained in a cubic surface by Lemma 3.9. We have $h^{0}\left(\mathcal{O}_{\mathbb{P}^{3}}(3) \otimes \mathcal{O}_{S}\right) \geqslant h^{0}\left(\mathcal{O}_{\mathbb{P}^{3}}(3)\right)=20$, because there is an exact sequence of the cohomology groups.

$$
0 \longrightarrow H^{0}\left(\mathcal{O}_{\mathbb{P}^{3}}(3) \otimes \mathcal{I}\right) \longrightarrow H^{0}\left(\mathcal{O}_{\mathbb{P}^{3}}(3)\right) \longrightarrow H^{0}\left(\mathcal{O}_{\mathbb{P}^{3}}(3) \otimes \mathcal{O}_{S}\right)
$$

By the Riemann-Roch theorem, we have

$$
20 \leqslant h^{0}\left(\mathcal{O}_{\mathbb{P}^{3}}(3) \otimes \mathcal{O}_{S}\right)=3 d-g+1+h^{1}\left(\mathcal{O}_{\mathbb{P}^{3}}(3) \otimes \mathcal{O}_{S}\right)=3 d-18+h^{1}\left(\mathcal{O}_{\mathbb{P}^{3}}(3) \otimes \mathcal{O}_{S}\right),
$$

which implies that $h^{1}\left(\mathcal{O}_{\mathbb{P}^{3}}(3) \otimes \mathcal{O}_{S}\right) \neq 0$, because $d \leqslant 12$ by Lemma 4.15. Then it follows from Theorem 2.19 that $19 \geqslant 3 d / 2+1 \geqslant h^{0}\left(\mathcal{O}_{\mathbb{P}^{3}}(3) \otimes \mathcal{O}_{S}\right) \geqslant 20$, which is a contradiction.

Lemma 4.25. The inequality $g \neq 16$ holds.

Proof. Suppose that $g=16$. Arguing as in the proof of Lemma 4.24, and keeping in mind that $d \leqslant 11$ by Lemma 4.15 , we see that $18 \geqslant 3 d / 2+1 \geqslant h^{0}\left(\mathcal{O}_{\mathbb{P}^{3}}(3) \otimes \mathcal{O}_{S}\right) \geqslant 20$, which is a contradiction.

Therefore, we see that $g=10$. Then $d \geqslant 9$ by Theorem 2.20 ,

Lemma 4.26. The inequality $d \neq 9$ holds.

Proof. Suppose that $d=9$. It follows from Lemma 3.7 that $h^{0}\left(\mathcal{O}_{\mathbb{P}^{3}}(2) \otimes \mathcal{I}\right) \neq 1$, which implies that $S=F_{1} \cap F_{2}$, where $F_{1}$ and $F_{2}$ are cubic surfaces in $\mathbb{P}^{3}$ (see [22, Example 6.4.3])

The group $\bar{G}$ cannot act non-trivially on the pencil generated by $F_{1}$ and $F_{2}$, which implies that the surfaces $F_{1}$ and $F_{2}$ must be $\bar{G}$-invariant. The latter is impossible by Lemma 3.7 .

Lemma 4.27. The inequality $d \neq 10$ holds.

Proof. Suppose that $d=10$. Then $q=4$ by Lemma 4.15, which is impossible by Lemma 4.17.

Thus we see that $d \geqslant 11$. Then $q=0$ and $d=11$ by Lemma 4.15,

Take a subgroup $\bar{F} \subset \bar{G}$ such that $\bar{F} \cong \mathrm{A}_{5}$ and the embedding $\bar{F} \subset \bar{G}$ is non-standard (see Definition 3.1). Then there is an $\bar{F}$-invariant twisted cubic curve $C \subset \mathbb{P}^{3}$ by Lemma 3.6.

Let $R$ be the quartic surface in $\mathbb{P}^{3}$ that is swept out by the lines that are tangent to $C$. Then the surface $R$ is $\bar{F}$-invariant, and the curve $S$ is not contained in the surface $R$, because $q=0$. Put $\Sigma=R \cap S$. We have $\Sigma=\Sigma_{1} \cup \ldots \cup \Sigma_{r}$, where $\Sigma_{i}$ is a $\bar{F}$-orbit. Hence, we have

$$
44=R \cdot S=\sum_{i=1}^{r} a_{i}\left|\Sigma_{i}\right|
$$


for some positive integers $a_{1}, \ldots, a_{r}$. We may assume that $\left|\Sigma_{1}\right| \geqslant \ldots \geqslant\left|\Sigma_{r}\right|$. But $\left|\Sigma_{i}\right| \in$ $\{12,20,30,60\}$ for every $i \in\{1, \ldots, r\}$ by Lemma 2.9. Thus, we see that $\left|\Sigma_{1}\right|=20$.

Let $O \in \Sigma_{1}$ be a point, and let $\bar{F}_{O} \subset \bar{F}$ be the stabilizer of the point $O$. Then $\bar{F}_{O} \cong \mathbb{Z}_{3}$.

Let $\Gamma$ be the $\bar{G}$-orbit of the point $O$, and let $\bar{G}_{O} \subset \bar{G}$ be the stabilizer of the point $O$. Then the order of the group $\bar{G}_{O}$ must divide 18. Moreover, the group $\bar{G}_{O}$ is cyclic by Lemma 2.9 , which implies that $\bar{G}_{O} \cong \mathbb{Z}_{3}$. Hence, we see that $|\Gamma|=120$, which contradicts Lemma 2.10.

The obtained contradiction completes the proof of Theorem 4.3 .

\section{Segre CubiC}

Let $G \subset \mathrm{SL}_{5}(\mathbb{C})$ be a subgroup such that $G \cong \mathrm{A}_{6}$. Let $\phi: \mathrm{SL}_{5}(\mathbb{C}) \rightarrow \operatorname{Aut}\left(\mathbb{P}^{4}\right)$ be the natural projection. Put $W=\mathbb{C}^{5}$ and $\bar{G}=\phi(G) \subset \operatorname{Aut}\left(\mathbb{P}^{4}\right)$. Then the space $W$ is an irreducible representation of the group $G \cong \bar{G} \cong \mathrm{A}_{6}$, and there is a unique cubic hypersurface $X \subset \mathbb{P}^{4}$ that is $\bar{G}$-invariant. Let us identify $X$ with a complete intersection in $\mathbb{P}^{5}$ that is given by the equation

$$
\sum_{i=0}^{5} x_{i}=\sum_{i=0}^{5} x_{i}^{3}=0 \subset \mathbb{P}^{5} \cong \operatorname{Proj}\left(\mathbb{C}\left[x_{0}, x_{1}, x_{2}, x_{3}, x_{4}, x_{5}\right]\right),
$$

and let us identify $\bar{G}$ with a subgroup of the group Aut $(X)$ (cf. Example 1.19).

Let $O \in X$ be a point, and let $\bar{F} \subset \bar{G}$ be its stabilizer.

Remark 5.1. Let $\tilde{T}$ be the affine tangent space to $\mathbb{P}^{4}$ at the point $O \in \mathbb{P}^{4}$. Then $\bar{F}$ naturally acts on the space $\tilde{T}$. Let us consider $W$ as a representation of the group $\bar{F}$. One has

$$
\tilde{T} \cong W / W_{O} \otimes W_{O}^{*}
$$

where $W_{O}$ is the one-dimensional subrepresentation of $\bar{F}$ that corresponds to the point $O \in \mathbb{P}^{4}$.

Let $\Sigma$ be the $\bar{G}$-orbit of the point $O \in X$.

Lemma 5.2. Suppose that $|\Sigma| \leqslant 15$. Then $|\Sigma| \in\{10,15\}$.

Proof. One has $|\Sigma| \neq 1$, because $W$ is an irreducible representation of the group $\bar{G}$. Hence $|\Sigma| \in\{6,10,15\}$ by Lemma 3.3. Suppose that $|\Sigma|=6$. Then $\bar{F} \cong \mathrm{A}_{5}$ by Lemma 3.3 ,

Let us consider $W$ as a representation of the group $\bar{F}$. Then $W$ is reducible and $W \cong W_{t} \oplus W_{4}$, where $W_{t}$ and $W_{4}$ are the trivial and a four-dimensional representations of the group $\bar{F}$, respectively. The embedding $\bar{F} \subset \bar{G}$ is standard (see Definition [3.1), because $W$ is reducible.

Note that $W_{t}$ is the only one-dimensional subrepresentation of the representation $W$, because the representation $W_{4}$ is irreducible. Hence, the set $\Sigma$ must contain a point in $\mathbb{P}^{5}$ that is given by $x_{0}=\ldots=x_{4}=-x_{5} / 5$, which is impossible, because this point does not belong to the hypersurface $X \subset \mathbb{P}^{5}$.

Let $T$ be the affine tangent space to $X$ at the point $O$. Then $\bar{F}$ naturally acts on $T$.

Lemma 5.3. Suppose that $|\Sigma|=10$. Then $\Sigma=\operatorname{Sing}(X)$ and $\bar{F} \cong\left(\mathbb{Z}_{3} \times \mathbb{Z}_{3}\right) \rtimes \mathbb{Z}_{4}$. Moreover, $T$ is an irreducible four-dimensional representation of the group $\bar{F}$.

Proof. One has $\bar{F} \cong\left(\mathbb{Z}_{3} \times \mathbb{Z}_{3}\right) \rtimes \mathbb{Z}_{4}$ by Lemma 3.3.

Up to conjugation, the group $\bar{G}$ has a unique subgroup that is isomorphic to $\bar{F}$, which implies that we may assume that $\bar{F}$ fixes the point $[1:-1: 1:-1: 1:-1] \in \operatorname{Sing}(X)$.

Let us consider $W$ as a representation of the group $\bar{F}$, and let $W_{1} \subset W$ be the one-dimensional subrepresentation of the group $\bar{G}$ that corresponds to the point $[1:-1: 1:-1: 1:-1]$. Then $W \cong W_{1} \oplus W_{4}$, where $W_{4}$ is some four-dimensional representation of the group $\bar{F}$.

Let $\chi_{1}$ and $\chi$ be the characters of the representations $W_{1}$ and $W$, respectively. The values of the characters $\chi_{1}$ and $\chi$ and the structure of the subgroup $\bar{F}$ are given in the following table: 


\begin{tabular}{|c|c|c|c|c|c|}
\hline & {$[4,2]$} & {$[3,3]$} & {$[3,1,1,1]$} & {$[2,2,1,1]$} & $e$ \\
\hline$\#$ & 18 & 4 & 4 & 9 & 1 \\
\hline$\chi$ & -1 & -1 & 2 & 1 & 5 \\
\hline$\chi_{1}$ & -1 & 1 & 1 & 1 & 1 \\
\hline
\end{tabular}

where we use notation similar to the ones used in the proof of Lemma 3.7.

We see that $W_{4}$ is an irreducible representation of the group $\bar{F}$. Thus, without loss of generality, we may assume that $O=[1:-1: 1:-1: 1:-1]$. Then $T \cong W_{4} \otimes W_{1}^{*}$ by Remark 5.1 , which implies that $T$ is an irreducible representation of the group $\bar{F}$.

Let $\Gamma$ be the $\bar{G}$-orbit of the point $[0: 0: 0: 0: 1:-1] \in X \backslash \operatorname{Sing}(X)$. Then $|\Gamma|=15$.

Lemma 5.4. Suppose that $|\Sigma|=15$. Then $\Sigma=\Gamma$, and $\bar{F} \cong \mathrm{S}_{4}$. Moreover, $T$ is an irreducible three-dimensional representation of the group $\bar{F}$.

Proof. One has $\bar{F} \cong \mathrm{S}_{4}$ by Lemma 3.3 . Up to conjugation, the group $\bar{G}$ contains exactly two non-conjugate subgroups that are isomorphic to the group $\bar{F} \cong \mathrm{S}_{4}$ (see Lemma 3.3).

Let us consider $W$ as a representation of the group $\bar{F}$. Then $W$ contains some one-dimensional subrepresentation $U$ of the group $\bar{F}$ that correspond to the point $O \in X$.

Let $W_{t}$ be the trivial one-dimensional representations of the group $\bar{F}$, and let $W_{1}$ be the nontrivial one-dimensional representations of the group $\bar{F}$. Then either $U \cong W_{t}$ or $U \cong W_{1}$.

Let $\chi$ and $\chi_{1}$ be the characters of $W$ and $W_{1}$, respectively.

Suppose that $\mathrm{S}_{4} \cong \bar{F} \subset \bar{G} \cong \mathrm{A}_{6}$ is a non-standard embedding. The values of $\chi_{1}$ and $\chi$ and the structure of the subgroup $\bar{F}$ are given in the following table:

\begin{tabular}{|c|c|c|c|c|c|}
\hline & {$[4,2]$} & {$[3,3]$} & {$[2,2,1,1]$} & {$[2,2,1,1]$} & $e$ \\
\hline$\#$ & 6 & 8 & 3 & 6 & 1 \\
\hline$\chi$ & -1 & -1 & 1 & 1 & 5 \\
\hline$\chi_{1}$ & -1 & 1 & 1 & -1 & 1 \\
\hline
\end{tabular}

where we use notation similar to the ones used in the proof of Lemma 3.7, and we divide the elements of type $[2,2,1,1]$ that are contained in the subgroup $\bar{F}$ into two classes with respect to the values of the character $\chi_{1}$. We see that $W$ contains no one-dimensional subrepresentations.

Thus $\mathrm{S}_{4} \cong \bar{F} \subset \bar{G} \cong \mathrm{A}_{6}$ is a standard embedding. The values of $\chi_{1}$ and $\chi$ and the structure of the subgroup $\bar{F}$ are given in the following table:

\begin{tabular}{|c|c|c|c|c|c|}
\hline & {$[4,2]$} & {$[3,1,1,1]$} & {$[2,2,1,1]$} & {$[2,2,1,1]$} & $e$ \\
\hline$\#$ & 6 & 8 & 3 & 6 & 1 \\
\hline$\chi$ & -1 & 2 & 1 & 1 & 5 \\
\hline$\chi_{1}$ & -1 & 1 & 1 & -1 & 1 \\
\hline
\end{tabular}

which implies that $W \cong W_{t} \oplus W_{1} \oplus W_{3}$, where $W_{3}$ is an irreducible three-dimensional representation of the group $\bar{F}$. Hence $T$ is an irreducible representation of the group $\bar{F}$ by Remark 5.1 .

Suppose that $\Sigma \neq \Gamma$. Then $[1: 1: 1: 1:-2:-2] \in \Sigma$. But $[1: 1: 1: 1:-2:-2] \notin X$, which gives a contradiction.

Let $H$ be a general hyperplane section of the cubic $X \subset \mathbb{P}^{4}$. Then the $\bar{G}$-invariant subgroup of the group $\mathrm{Cl}(X)$ is generated by $H$ (see Example 1.19). The main purpose of this section is to prove

Theorem 5.5. The threefold $X$ is $\bar{G}$-birationally superrigid and $\operatorname{Bir}^{\bar{G}}(X) \cong \mathrm{S}_{6}$. 
By Corollary 1.12 , the isomorphism $\operatorname{Bir}^{\bar{G}}(X) \cong \mathrm{S}_{6}$ follows from $\bar{G}$-birational superrigidity of $X$ and classification of primitive subgroups in $\mathrm{SL}_{5}(\mathbb{C})$ (see [17]). In the remaining part of this section, we will prove that $X$ is $\bar{G}$-birationally superrigid.

Suppose that $X$ is not $\bar{G}$-birationally superrigid. Let us derive a contradiction. It follows from [12, Theorem 4.2] or [7, Theorem 1.4.1] that there is a (non-empty) $\bar{G}$-invariant linear system $\mathcal{M}$ on $X$ such that $\mathcal{M}$ does not have fixed components, and $(X, \lambda \mathcal{M})$ is not canonical, where $\lambda \in \mathbb{Q}$ such that $\lambda \mathcal{M} \sim \mathbb{Q}-K_{X}$. By Corollary 2.3 there is $\mu \in \mathbb{Q}$ such that $\mu<2 \lambda$ and $(X, \mu \mathcal{M})$ is strictly $\log$ canonical. Let $S \subset X$ be a minimal center in $\mathbb{L} \mathbb{C} \mathbb{S}(X, \mu \mathcal{M})$. Then it follows from Lemma 2.7 that $S \cap g(S) \neq \varnothing$ if and only if $S=g(S)$ for every $g \in \bar{G}$. Moreover, the center $S$ is not a surface, since $\mathcal{M}$ has no fixed components.

By Lemma 2.13, there is a $\bar{G}$-invariant effective $\mathbb{Q}$-divisor $D$ on $X$ such that the set $\mathbb{L} \mathbb{C} S(X, D)$ consists of irreducible components of the $\bar{G}$-orbit of $S$, the log pair $(X, D)$ is log canonical and $D \sim \mathbb{Q}-\delta K_{X}$ for some $\delta \in \mathbb{Q}$ such that $0<\delta<2$. Arguing as in the proof of [10, Lemma 2.11], we see that we can replace $D$ by $(\mu-\epsilon) \mathcal{M}+\epsilon^{\prime} \mathcal{H}$, where $\epsilon \in \mathbb{Q}_{>0} \ni \epsilon^{\prime}$ such that $\epsilon \ll 1$ and $\epsilon^{\prime} \ll 1$, and $\mathcal{H}$ is a $\bar{G}$-invariant linear system such that $\mathcal{H} \sim-n K_{X}$ holds for some $n \gg 0$, the base locus of the linear system $\mathcal{H}$ coincides with $\mathbb{L} \mathbb{C S}(X, D)$, and $\delta=(\mu-\epsilon) / \lambda+\epsilon^{\prime} n$. Thus, without loss of generality, we can replace $D$ by $\mu \mathcal{M}$ (cf. Lemma 2.13). Therefore, without loss of generality, we may assume that the set $\mathbb{L} \mathbb{C S}(X, \mu \mathcal{M})$ also consists of irreducible components of the $\bar{G}$-orbit of $S$.

Let $\mathcal{I}$ be the multiplier ideal sheaf of $(X, \mu \mathcal{M})$, and let $\mathcal{L}$ be the subscheme given by $\mathcal{I}$. Then

$$
h^{0}\left(\mathcal{O}_{\mathcal{L}} \otimes \mathcal{O}_{X}(2 H)\right)=h^{0}\left(\mathcal{O}_{X}(2 H)\right)-h^{0}\left(\mathcal{O}_{X}(2 H) \otimes \mathcal{I}\right)=15-h^{0}\left(\mathcal{O}_{X}(2 H) \otimes \mathcal{I}\right)
$$

by Theorem 2.4 .

Lemma 5.7. The center $S$ is not a singular point of the threefold $X$.

Proof. Suppose that $S$ is a singular point of the threefold $X$. Then $\operatorname{LCS}(X, \mu \mathcal{M})=\operatorname{Sing}(X)$, because $\bar{G}$ acts transitively on the set $\operatorname{Sing}(X)$. Recall that $|\operatorname{Sing}(X)|=10$.

Let $\bar{F} \subset \bar{G}$ be a stabilizer of the point $S$. Then $\bar{F} \cong\left(\mathbb{Z}_{3} \times \mathbb{Z}_{3}\right) \rtimes \mathbb{Z}_{4}$ by Lemma 5.3 .

Let $\pi: U \rightarrow X$ be a blow up of the points $\operatorname{Sing}(X)$, and let $E_{1}, \ldots, E_{10}$ be the $\pi$-exceptional divisors. Then there is a positive rational number $m$ such that

$$
K_{U}+\mu \overline{\mathcal{M}}+(m-1) \sum_{i=1}^{10} E_{i} \sim_{\mathbb{Q}} \pi^{*}\left(K_{X}+\mu \mathcal{M}\right),
$$

where $\overline{\mathcal{M}}$ is the proper transforms of the linear system $\mathcal{M}$ on the variety $U$.

Note that it follows from [7, Theorem 1.7.20] that $m \geqslant 1$ (cf. [13, Theorem 3.10]).

We may assume that $\pi\left(E_{1}\right)=S$. There is a natural homomorphism $v: \bar{F} \rightarrow \operatorname{Aut}\left(E_{1}\right)$. Then $\operatorname{lct}\left(E_{1}, v(\bar{F})\right) \geqslant 1$ by Lemmas 2.16 and 5.3 , because $E_{1} \cong \mathbb{P}^{1} \times \mathbb{P}^{1}$.

Let us show that $\operatorname{lct}\left(E_{1}, v(\bar{F})\right)<1$ to derive a contradiction.

One can easily check that there exists a two-dimensional linear subspace $\Pi \subset \mathbb{P}^{4}$ such that $|\Pi \cap \operatorname{Sing}(X)|=4$ and $\Pi \subset X$ (see [23, Section 3.2]). Let $C$ be a general conic in $\Pi$ that contains $\Pi \cap \operatorname{Sing}(X)$. Then $C$ is not contained in the base locus of the linear system $\mathcal{M}$, and $C$ is irreducible. Let $\bar{C}$ be a proper transform of the curve $C$ on the threefold $U$. Then

$$
\frac{8}{\mu}-4 \frac{m}{\mu}>\frac{4}{\lambda}-4 \frac{m}{\mu}=\overline{\mathcal{M}} \cdot \bar{C} \geqslant 0
$$

which implies that $m<2$. In particular, the $\log$ pair $\left(U, \mu \overline{\mathcal{M}}+(m-1) \sum_{i=1}^{10} E_{i}\right)$ is not Kawamata $\log$ terminal along $E_{1}$. Hence $\operatorname{lct}\left(E_{1}, v(\bar{F})\right)<1$ by Theorem 2.6.

Lemma 5.8. The center $S$ is a curve. 
Proof. Suppose that $S$ is a point. Then $S \notin \operatorname{Sing}(X)$ by Lemma 5.8. It follows from (5.6) that $|\operatorname{LCS}(X, \mu \mathcal{M})| \leqslant 15$, so that $\operatorname{LCS}(X, \mu \mathcal{M})$ is the $\bar{G}$-orbit of the point $[0: 0: 0: 0: 1:-1]$ by Lemma 5.2. There are 15 two-dimensional linear subspaces $\Pi_{1}, \ldots, \Pi_{15}$ in $\mathbb{P}^{4}$ such that $\mid \Pi_{i} \cap$ $\operatorname{LCS}(X, \mu \mathcal{M}) \mid=6$ and $X \cap \Pi_{i}=L_{i}^{1}+L_{i}^{2}+L_{i}^{3}$ for any $i \in\{1, \ldots, 15\}$, where $L_{i}^{j}$ is a line such that

$$
\left(\left(L_{i}^{1} \cap L_{i}^{2}\right) \cup\left(L_{i}^{1} \cap L_{i}^{3}\right) \cup\left(L_{i}^{2} \cap L_{i}^{3}\right)\right) \bigcap \operatorname{LCS}(X, \mu \mathcal{M})=\varnothing
$$

and $L_{i}^{j}=L_{r}^{s}$ if and only if $(i, j)=(r, s)$. Note that the curve $\sum_{i=1}^{15}\left(L_{i}^{1}+L_{i}^{2}+L_{i}^{3}\right)$ is a $\bar{G}$-orbit of the line $L_{1}^{1}$. Without loss of generality, we may assume that $S \in L_{1}^{1}$.

Let $M_{1}$ and $M_{2}$ be general surfaces in the linear system $\mathcal{M}$. Put

$$
M_{1} \cdot M_{2}=\gamma \sum_{i=1}^{15}\left(L_{i}^{1}+L_{i}^{2}+L_{i}^{3}\right)+\Omega
$$

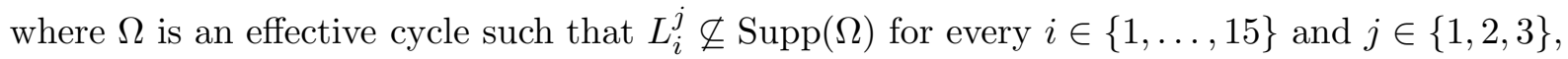
and $\gamma$ is a non-negative rational number. Put $m=\operatorname{mult}_{S}\left(M_{1} \cdot M_{2}\right)$, and let $D$ be a general surface in $|H|$ that contains the lines $L_{1}^{1}, L_{1}^{2}$ and $L_{1}^{3}$. Then

$$
\frac{12}{\lambda^{2}}-3 \gamma=D \cdot\left(\Omega+\gamma \sum_{i=2}^{15}\left(L_{i}^{1}+L_{i}^{2}+L_{i}^{3}\right)\right) \geqslant 6(m-\gamma),
$$

which implies that $\gamma \geqslant 2 m-4 / \lambda^{2}$. Therefore, we see that

$$
\frac{12}{\lambda^{2}}=H \cdot M_{1} \cdot M_{2}=45 \gamma+H \cdot \Omega \geqslant 45 \gamma \geqslant 45\left(2 m-\frac{4}{\lambda^{2}}\right),
$$

which implies that $m \leqslant 32 /\left(15 \lambda^{2}\right)$. In particular, we see that

$$
\operatorname{mult}_{S}(\mathcal{M}) \leqslant \sqrt{m} \leqslant \frac{2 \sqrt{2}}{\sqrt{15} \lambda}<\frac{3}{2 \lambda}<\frac{3}{\mu} .
$$

Let $\pi: U \rightarrow X$ be a blow up of the point $S$, and let $E$ be the $\pi$-exceptional divisor. Then

$$
K_{U}+\mu \overline{\mathcal{M}}+\left(\mu \operatorname{mult}_{S}(\mathcal{M})-2\right) E \sim_{\mathbb{Q}} \pi^{*}\left(K_{X}+\mu \mathcal{M}\right)
$$

where $\overline{\mathcal{M}}$ is the proper transforms of $\mathcal{M}$ on the variety $U$.

Let $\bar{F} \subset \bar{G}$ be a stabilizer of the point $S$. Then $\bar{F} \cong \mathrm{S}_{4}$ by Lemma 5.4, and there is a natural homomorphism $v: \bar{F} \rightarrow \operatorname{Aut}(E)$. Note that $v$ is a monomorphism by Lemma 5.4 .

There is an irreducible proper subvariety $C \subsetneq E \cong \mathbb{P}^{2}$ such that

$$
g(C) \in \mathbb{L} \mathbb{C S}\left(U, \mu \overline{\mathcal{M}}+\left(\mu \operatorname{mult}_{S}(\mathcal{M})-2\right) E\right)
$$

for every $g \in v(\bar{F})$. Then $C$ is a curve by Theorem 2.5 and Lemma 5.4.

Let $\bar{M}_{1}$ and $\bar{M}_{2}$ be general surfaces in $\overline{\mathcal{M}}$. Then it follows from [13, Theorem 3.1] that

$$
\operatorname{mult}_{g(C)}\left(\bar{M}_{1} \cdot \bar{M}_{2}\right) \geqslant \frac{4}{\mu^{2}}\left(3-\mu \operatorname{mult}_{S}(\mathcal{M})\right) \text {. }
$$

for every $g \in v(\bar{F})$. Let $\delta$ be the degree in $E \cong \mathbb{P}^{2}$ of the $v(\bar{F})$-orbit of the curve $C$. Then

$$
\frac{128}{15 \mu^{2}} \geqslant \frac{32}{15 \lambda^{2}} \geqslant m \geqslant \operatorname{mult}_{S}^{2}(\mathcal{M})+\delta \operatorname{mult}_{C}\left(\bar{M}_{1} \cdot \bar{M}_{2}\right) \geqslant \operatorname{mult}_{S}^{2}(\mathcal{M})+\frac{8}{\mu^{2}}\left(3-\mu \operatorname{mult}_{S}(\mathcal{M})\right),
$$

because $\delta \geqslant 2$ by Lemma [5.4. The latter value is greater than $9 / \mu^{2}$, which can be easily seen using the elementary properties of quadratic forms and (5.9). The obtained contradiction completes the proof.

By Theorem 2.8, the curve $S$ is a smooth curve in $\mathbb{P}^{4}$ of degree $d$ and genus $g \leqslant d$. Put $q=h^{0}\left(\mathcal{O}_{X}(2 H) \otimes \mathcal{I}\right)$, let $Z$ be the $\bar{G}$-orbit of the curve $S$, let $r$ be the number of irreducible components of the curve $Z$. 
Lemma 5.10. The equality $r(2 d-g+1)=15-q$ holds.

Proof. The equality follows from (5.6) and the Riemann-Roch theorem, because $d \geqslant g$.

Corollary 5.11. The inequality $g \leqslant 14$ holds.

Note that $Z$ is not contained in a hyperplane in $\mathbb{P}^{4}$, since $W$ is an irreducible $\bar{G}$-representation.

Lemma 5.12. The equality $r=1$ holds.

Proof. Suppose that $r \geqslant 2$. Then $r \leqslant 15$ by Lemma 5.10, which implies that $r \in\{6,10,15\}$ by Lemma 3.3. If $q=0$, then $2 d-g+1=1$ by Lemma 5.10, which is impossible, because $g \leqslant d$.

We have $q \geqslant 1$. Then $r(g+1) \leqslant 14$ and $g \leqslant 1$. If $g=0$, then $r(2 d+1) \leqslant 14$ by Lemma 5.10 , which implies a contradictory inequality $d \leqslant 0$. We see that $g=1$. Thus $2 r d \leqslant 14$ by Lemma 5.10, which implies that $d=1$ and $g=0$, that contradicts the equality $g=1$.

There is a natural monomorphism $\theta: \bar{G} \rightarrow \operatorname{Aut}(S)$ (see Lemma 5.2).

Lemma 5.13. The equality $g=10$ holds.

Proof. The required assertion follows from Lemmas 5.10 and 2.10.

The equality $g=10$ and Lemma 5.10 imply that $d \leqslant 12$.

Lemma 5.14. The equality $q=0$ holds.

Proof. Let $\bar{\Psi} \subset \bar{G}$ be a subgroup such that $\bar{\Psi} \cong \mathrm{A}_{5}$ and the embedding $\mathrm{A}_{5} \cong \bar{\Psi} \subset \bar{G} \cong \mathrm{A}_{6}$ is standard. There is a $\bar{\Psi}$-invariant hyperplane section $H \subset X$. Note that $S \not \subset H$. We have $|H \cap S| \leqslant d \leqslant 12$, which implies that $|H \cap S|=12$ by Lemma 2.9 , because $H \cap S$ is $\bar{\Psi}$-invariant. Then $q=0$ by Lemma 5.10 .

Let $Q$ be the $\bar{G}$-invariant quadric in $\mathbb{P}^{4}$ (cf. Example 1.18). Then $S \not \subset Q$, because $q=0$.

Put $\Delta=Q \cap S$. Then $|\Delta| \leqslant 24$. Let $\bar{\Psi} \subset \bar{G}$ be a stabilizer of a point in $\Delta$. Then

$$
|\bar{\Psi}| \geqslant \frac{|\bar{G}|}{|\Delta|} \geqslant \frac{360}{24}>6
$$

which is impossible by Lemma 2.9 .

The obtained contradiction completes the proof of Theorem 5.5 .

\section{QuADRIC THREEFOLD}

Let $G \subset \mathrm{SL}_{5}(\mathbb{C})$ be a subgroup such that $G \cong \mathrm{A}_{6}$, and let $\phi: \mathrm{SL}_{5}(\mathbb{C}) \rightarrow \operatorname{Aut}\left(\mathbb{P}^{4}\right)$ be the natural projection. Put $W=\mathbb{C}^{5}$ and $\bar{G}=\phi(G)$. Then there is a smooth quadric hypersurface $Q \subset \mathbb{P}^{4}$ that is $\bar{G}$-invariant. Let us identify $Q$ with a smooth complete intersection in $\mathbb{P}^{5}$ that is given by the equation

$$
\sum_{i=0}^{5} x_{i}=\sum_{i=0}^{5} x_{i}^{2}=0 \subset \mathbb{P}^{5} \cong \operatorname{Proj}\left(\mathbb{C}\left[x_{0}, x_{1}, x_{2}, x_{3}, x_{4}, x_{5}\right]\right),
$$

and let us identify $\bar{G}$ with a subgroup of the group $\operatorname{Aut}(Q)$ (cf. Example 1.18).

Let $O \in Q$ be a point, let $\bar{F} \subset \bar{G}$ be its stabilizer, and let $\Sigma$ be its $\bar{G}$-orbit.

Lemma 6.1. Suppose that $|\Sigma| \leqslant 30$. Then $|\Sigma|=30$, and there exists a cubic hypersurface $X \subset \mathbb{P}^{4}$ such that $\Sigma \subset X$ and $Q \not \subset X$.

Proof. One has $|\Sigma| \neq 1$, because $W$ is an irreducible representation of the group $\bar{G}$. Then $|\Sigma| \in\{6,10,15,20,30\}$ by Lemma 3.3. Arguing as in the proof of Lemmas 4.2, 5.2, 5.3 and 5.4, we see that $|\Sigma| \in\{20,30\}$. 
Let us consider $W$ as a representation of the group $\bar{F}$. Then $W$ contains some one-dimensional subrepresentation $U$ of the group $\bar{F}$ corresponding to the point $O \in Q$.

Suppose that $|\Sigma|=20$. Then $\bar{F} \cong\left(\mathbb{Z}_{3} \times \mathbb{Z}_{3}\right) \rtimes \mathbb{Z}_{2}$ by Lemma 3.3 .

Let $W_{t}$ and $W_{1}$ be the trivial one-dimensional and the non-trivial one-dimensional representations of $\bar{F}$ (see the proof of Lemma 5.3), respectively. Then either $U \cong W_{t}$ or $U \cong W_{1}$.

Let $\chi$ and $\chi_{1}$ be the characters of the representations $W$ and $W_{1}$, respectively. The values of the characters $\chi_{1}$ and $\chi$ and the structure of the subgroup $\bar{F}$ are given in the following table:

\begin{tabular}{|c|c|c|c|c|}
\hline & {$[3,3]$} & {$[3,1,1,1]$} & {$[2,2,1,1]$} & $e$ \\
\hline$\#$ & 4 & 4 & 9 & 1 \\
\hline$\chi$ & -1 & 2 & 1 & 5 \\
\hline$\chi_{1}$ & 1 & 1 & -1 & 1 \\
\hline
\end{tabular}

where we use notation similar to the ones used in the proofs of Lemmas 3.7, 5.3 and 5.4,

We see that $U \cong W_{1}$. Thus $[1:-1: 1:-1: 1:-1] \in \Sigma$. But $[1:-1: 1:-1: 1:-1] \notin Q$.

Therefore, we see that $|\Sigma|=30$. Then $\bar{F} \cong \mathrm{A}_{4}$ by Lemma 3.3 . The embedding $\mathrm{A}_{4} \cong \bar{F} \subset$ $\bar{G} \cong \mathrm{A}_{6}$ must be standard, because otherwise the representation $W$ would be an irreducible representation of the group $\bar{F}$ (cf. the proof of Lemma 5.4).

There are exactly two $\bar{F}$-invariant points in $Q$. These points form a subset

$$
\{[1: 1: 1: 1:-2+\sqrt{-2}:-2-\sqrt{-2}],[1: 1: 1: 1:-2-\sqrt{-2}:-2+\sqrt{-2}]\} \subset \Sigma .
$$

Let $X$ be the cubic threefold in $\mathbb{P}^{5}$ that is given by

$$
\sum_{i=0}^{5} x_{i}=\left(x_{0}-x_{1}\right)\left(x_{2}-x_{3}\right)\left(x_{4}-x_{5}\right)=0,
$$

let $P$ be the point $\left[1: \omega: \omega^{2}: 1: \omega: \omega^{2}\right] \in \mathbb{P}^{5}$, where $\omega$ is a non-trivial cube root of unity. Then $\Sigma \subset X \not \ngtr P \in Q$, which completes the proof.

The main purpose of this section is to prove the following result.

Theorem 6.2. The quadric threefold $Q$ is $\bar{G}$-birationally superrigid and $\operatorname{Bir}^{\bar{G}}(Q) \cong \mathrm{S}_{6}$.

By Corollary 1.12, the isomorphism $\operatorname{Bir}^{\bar{G}}(Q) \cong \mathrm{S}_{6}$ follows from $\bar{G}$-birational superrigidity of $Q$ and classification of primitive subgroups in $\mathrm{SL}_{5}(\mathbb{C})$ (see [17]). In the remaining part of this section, we will prove that $Q$ is $\bar{G}$-birationally superrigid.

Suppose that $Q$ is not $\bar{G}$-birationally superrigid. Arguing as in the proof of Theorem 5.5, we see that there is a $\bar{G}$-invariant effective $\mathbb{Q}$-divisor $D$ on $Q$ such that the set $\mathbb{L} \mathbb{C}(Q, D)$ consists of irreducible components of the $\bar{G}$-orbit of $S$, the log pair $(Q, D)$ is log canonical, and $D \sim_{\mathbb{Q}}-\delta K_{Q}$ for some positive rational number $\delta<2$, where $S$ is a minimal center in $\mathbb{L} \mathbb{C S}(Q, D)$ such that either $S$ is a point or a smooth curve.

Let $\mathcal{I}$ be the multiplier ideal sheaf of the $\log$ pair $(Q, D)$, let $\mathcal{L}$ be subscheme that is given by the ideal sheaf $\mathcal{I}$, and let $H$ be a general hyperplane section of the threefold $Q \subset \mathbb{P}^{3}$. Then

$$
h^{0}\left(\mathcal{O}_{\mathcal{L}} \otimes \mathcal{O}_{Q}(3 H)\right)=h^{0}\left(\mathcal{O}_{Q}(3 H)\right)-h^{0}\left(\mathcal{O}_{Q}(3 H) \otimes \mathcal{I}\right)=30-h^{0}\left(\mathcal{O}_{Q}(3 H) \otimes \mathcal{I}\right)
$$

by Theorem 2.4 ,

Lemma 6.4. The center $S$ is a curve.

Proof. The required assertion follows from (6.3) and Lemma 6.1. 
By Theorem 2.8, the curve $S$ is a smooth curve of degree $d$ and genus $g$ such that

$$
g \leqslant \frac{3 d+1}{2} .
$$

Put $q=h^{0}\left(\mathcal{O}_{Q}(3 H) \otimes \mathcal{I}\right)$, let $Z$ be the $\bar{G}$-orbit of the curve $S$, let $r$ be the number of irreducible components of the curve $Z$.

Lemma 6.6. The equality $r(3 d-g+1)=30-q$ holds.

Proof. The equality follows from (6.3) and the Riemann-Roch theorem, because $3 d \geqslant 2 g-1$.

Let $X$ be the cubic threefold in $\mathbb{P}^{5}$ that is given by $\sum_{i=0}^{5} x_{i}=\sum_{i=0}^{5} x_{i}^{3}=0$ (cf. Example1.19).

Lemma 6.7. Suppose that $q \geqslant 2$. Then $q \geqslant 5$.

Proof. Suppose that $2 \leqslant q \leqslant 4$. It follows from [15] that $\bar{G}$ acts trivially on the $q$-dimensional vector space $H^{0}\left(\mathcal{O}_{Q}(3 H) \otimes \mathcal{I}\right)$. But $Q \cap X$ is the only $\bar{G}$-invariant surface in $\left|\mathcal{O}_{Q}(3 H)\right|$.

Note that $Z$ is not contained in a hyperplane in $\mathbb{P}^{4}$, since $W$ is an irreducible $\bar{G}$-representation.

Lemma 6.8. The curve $S$ is irreducible.

Proof. Suppose that $r \geqslant 2$. By Lemma 3.3, either $r=6$, or $r \geqslant 10$.

Suppose that $q=0$. If $r=6$, then it follows from Lemma 6.6 that

$$
3 d-g+1=5 \text {. }
$$

From (6.5) and (6.9) we obtain that $g \leqslant 5$. Applying (6.9) we see that $d \leqslant 3$ and thus $g \leqslant 1$. Applying (6.9) once again, we obtain a contradiction. Therefore, we see that $r \geqslant 10$ and

$$
3 d-g+1 \leqslant 3
$$

by Lemma 6.6. By (6.5) and (6.10) we have $g \leqslant 3$. Applying (6.10) again, we obtain $d=1$ and thus $g=0$, which is incompatible with (6.10).

Suppose that $q=1$. Then $r(3 d-g+1)=29$ by Lemma 6.6, which is impossible by Lemma 3.3.

Therefore, we see that $q \geqslant 2$. Hence $q \geqslant 5$ by Lemma 6.7. It follows from Lemma 6.6 that $r(3 d-g+1) \leqslant 25$, which implies that $3 d-g \leqslant 3$. We have $g \leqslant 4$, because $2 g-1 \leqslant 3 d$. Thus $d \leqslant 2$ and $g=0$.

Applying Lemma 6.6, we get $d=1$ and $r=6$. Then $S$ is a line, and $Z$ is a union of six lines.

Let $\bar{\Psi} \subset \bar{G}$ be a stabilizer of the line $S$. Then $\bar{\Psi} \cong \mathrm{A}_{5}$ by Lemma 3.3 ,

Let us consider $W$ as a representation of the group $\bar{\Psi} \cong \mathrm{A}_{5}$. Then either $W$ is irreducible, or $W \cong W_{t} \oplus W_{4}$, where $W_{t}$ and $W_{4}$ are the trivial one-dimensional and the standard fourdimensional representations of the group $\bar{\Psi} \cong \mathrm{A}_{5}$, respectively. In both cases, the line $S$ can not be $\bar{\Psi}$-invariant.

We see that $r=1$. Hence $g \leqslant 30$ and $d \leqslant 19$ by (6.3), because $3 d \geqslant 2 g-1$.

Lemma 6.11. The equalities $d=12$ and $g=10$ hold.

Proof. Let $\Pi_{i} \subset Q$ be a hyperplane section that is cut out by $x_{i}=0$, where $i \in\{0, \ldots, 5\}$. Then $\Pi_{0} \cap \ldots \cap \Pi_{5}=\varnothing$, which implies that, without loss of generality, we may assume that $S \not \subset \Pi_{0}$.

Let $\bar{\Psi} \subset \bar{G}$ be a stabilizer of the surface $\Pi_{0}$. Then $\bar{\Psi} \cong \mathrm{A}_{5}$ and the embedding $\bar{\Psi} \subset \bar{G}$ must be standard. Hence we have

$$
19 \geqslant d=\Pi_{0} \cdot S \geqslant\left|\Pi_{0} \cap S\right|,
$$

which implies that $d=\Pi_{0} \cdot S=\left|\Pi_{0} \cap S\right|=12$ by Lemma 2.15, because $\Pi_{0} \cong \mathbb{P}^{1} \times \mathbb{P}^{1}$.

It follows from Theorem 2.20 that $g \leqslant 15$. Thus $g=10$ by Lemma 2.10 .

Thus, it follows from Lemma 6.6 that $q=3$, which is impossible by Lemma 6.7.

The obtained contradiction completes the proof of Theorem 6.2 . 


\section{Appendix A. Klein Cubic threefold}

Put $\bar{\Gamma}=\mathrm{PSL}_{2}\left(\mathbb{F}_{11}\right)$. Let $V$ be a Fano threefold with terminal singularities such that $V$ admits a non-trivial action of the group $\bar{\Gamma}$, and the $\bar{\Gamma}$-invariant subgroup of the group $\mathrm{Cl}(V)$ is $\mathbb{Z}$.

Example A.1. If $V$ is a smooth hypersurface in $\mathbb{P}^{4}$ that is given by the equation

$$
x_{0}^{2} x_{1}+x_{1}^{2} x_{2}+x_{2}^{2} x_{3}+x_{3}^{2} x_{4}+x_{4}^{2} x_{0}=0 \subset \mathbb{P}^{4} \cong \operatorname{Proj}\left(\mathbb{C}\left[x_{0}, x_{1}, x_{2}, x_{3}, x_{4}\right]\right),
$$

then $V$ is non-rational by [11, Theorem 0.12], and $\operatorname{Aut}\left(V_{3}\right) \cong \bar{\Gamma}$ by [1].

Example A.2 ([38, Example 2.9]). There is a non-trivial action of the group $\bar{\Gamma}$ on $\operatorname{Gr}(2,6)$, and $\operatorname{Pic}(\operatorname{Gr}(2,6))=\mathbb{Z}[H]$ for some very ample divisor $H$. The linear system $|H|$ gives an embedding $\zeta: \operatorname{Gr}(2,6) \rightarrow \mathbb{P}^{14}$, which induces a non-trivial action of the group $\bar{\Gamma}$ on $\mathbb{P}^{14}$. Put $V=\zeta(\operatorname{Gr}(2,6)) \cap \Pi$, where $\Pi$ is the unique $\bar{\Gamma}$-invariant linear subspace $\Pi \subset \mathbb{P}^{14}$ such that $\operatorname{dim}(\Pi)=9$. Then the variety $V$ is a smooth Fano threefold such that $\operatorname{Pic}(V) \cong \mathbb{Z}$ and $-K_{V}^{3}=14$ (see [25]). Furthermore, the variety $V$ admits a non-trivial action of the group $\bar{\Gamma}$.

Let $V_{3}$ and $V_{14}$ be the threefolds that are constructed in Examples A.1 and A.2, respectively.

Theorem A.3 ([38, Theorem 1.5]). There is a $\bar{\Gamma}$-equivariant birational map $\chi: V \rightarrow U$ such that either $U \cong V_{3}$ or $U \cong V_{14}$.

Remark A.4 (see [38, Remark 2.10]). The varieties $V_{3}$ and $V_{14}$ are birationally equivalent.

The main purpose of this section is to prove the following result (cf. [38, Remark 2.10]).

Theorem A.5. The varieties $V_{3}$ and $V_{14}$ are $\bar{\Gamma}$-birationally superrigid.

Corollary A.6. The birational map $\chi: V \rightarrow U$ in Theorem A.3 is biregular.

Corollary A.7. There exists no $\bar{\Gamma}$-equivariant birational map $V_{14}-\rightarrow V_{3}$.

Corollary A.8. Up to conjugation, the group $\operatorname{Bir}\left(V_{3}\right) \cong \operatorname{Bir}\left(V_{14}\right)$ contains exactly 2 subgroups that are isomorphic to the simple group $\mathrm{PSL}_{2}\left(\mathbb{F}_{11}\right)$.

Let us prove that $V_{14}$ is $\bar{\Gamma}$-birationally superrigid. Suppose that $V_{14}$ is not $\bar{\Gamma}$-birationally superrigid. There is a (non-empty) $\bar{\Gamma}$-invariant linear system $\mathcal{M}$ without fixed components such that $\lambda \mathcal{M} \sim_{\mathbb{Q}}-K_{V_{14}}$ for some $\lambda \in \mathbb{Q}_{>0}$. Then $\left(V_{14}, \lambda \mathcal{M}\right)$ is not canonical (see [7, Theorem 1.4.1]).

There is $\mu \in \mathbb{Q}$ such that $\mu<2 \lambda$ and $\left(V_{14}, \mu \mathcal{M}\right)$ is strictly $\log$ canonical.

Let $S \subset V_{14}$ be a minimal center in $\mathbb{L} \mathbb{C S}\left(V_{14}, \mu \mathcal{M}\right)$. Then $\operatorname{dim}(S) \in\{0,1\}$.

By Lemma 2.13, there is a $\bar{\Gamma}$-invariant effective $\mathbb{Q}$-divisor $D$ on the threefold $V_{14}$ such that the set $\mathbb{L} \mathbb{C S}\left(V_{14}, D\right)$ consists of irreducible components of the $\Gamma$-orbit of $S$, and $D \sim_{\mathbb{Q}}-\epsilon K_{V_{14}}$, where $\epsilon$ is a positive rational number such that $\epsilon<2$.

Let $\mathcal{I}$ be the multiplier ideal sheaf of the log pair $\left(V_{14}, D\right)$, and let $\mathcal{L}\left(V_{14}, D\right)$ be the $\log$ canonical singularities subscheme of the $\log$ pair $\left(V_{14}, D\right)$. Then it follows from Theorem 2.4 that

(A.9) $h^{0}\left(\mathcal{O}_{\mathcal{L}\left(V_{14}, D\right)} \otimes \mathcal{O}_{V_{14}}(H)\right)=h^{0}\left(\mathcal{O}_{V_{14}}(H)\right)-h^{0}\left(\mathcal{O}_{V_{14}}(H) \otimes \mathcal{I}\right)=10-h^{0}\left(\mathcal{O}_{V_{14}}(H) \otimes \mathcal{I}\right)$, where $H \in\left|-K_{V_{14}}\right|$.

Lemma A.10. The equality $\operatorname{dim}(S)=0$ is impossible.

Proof. Suppose that $\operatorname{dim}(S)=0$. Let $\bar{F} \subset \bar{\Gamma}$ be a stabilizer of a point in $\operatorname{LCS}\left(V_{14}, D\right)$. Then $\left|\operatorname{LCS}\left(V_{14}, D\right)\right| \leqslant 10$ by (A.9) . Thus, we see that $|\bar{F}| \geqslant|\bar{\Gamma}| / 10=66$. Hence, we must have $\operatorname{LCS}\left(V_{14}, D\right)=S$ and $\bar{F}=\bar{\Gamma}$, because there are no proper subgroups of $\bar{\Gamma}$ of order greater than 60 (see [15]).

The action of $\bar{\Gamma}$ on the tangent space to $V_{14}$ at the point $S$ gives a faithful three-dimensional representation of the group $\bar{\Gamma}$, which does not exist (see [15]). 
It follows from Theorem 2.8 that $S$ is a smooth curve of genus $g$ such that $2 g-2<S \cdot H$.

Lemma A.11. The curve $S$ is $\bar{\Gamma}$-invariant.

Proof. Let $Z$ be the $\bar{\Gamma}$-orbit of the curve $S$. Then $Z=\mathcal{L}\left(V_{14}, D\right)$, because $\left(V_{14}, D\right)$ is $\log$ canonical.

Suppose that $S \neq Z$. Let $r$ be the number of irreducible components of $Z$. Then $r \geqslant 11$, because there is no nontrivial homomorphism $\bar{\Gamma} \rightarrow \mathrm{S}_{r}$ if $1<r \leqslant 10$.

Using (A.9) and the Riemann-Roch theorem, we see that

$$
10 \geqslant 10-h^{0}\left(\mathcal{O}_{V_{14}}(H) \otimes \mathcal{I}\right)=h^{0}\left(\mathcal{O}_{\mathcal{L}\left(V_{14}, D\right)} \otimes \mathcal{O}_{V_{14}}(H)\right)=r(S \cdot H-g+1) \geqslant r,
$$

because $\mathcal{L}\left(V_{14}, D\right)=Z$ and $2 g-2<S \cdot H$. Thus, we see that $r \leqslant 10$.

Therefore, there is a natural homomorphism $\theta: \bar{\Gamma} \rightarrow \operatorname{Aut}(S)$.

Lemma A.12. The homomorphism $\theta$ is a monomorphism.

Proof. Suppose that $\theta$ is not a monomorphism. Then $\operatorname{ker}(\theta)=\bar{\Gamma}$, because $\bar{\Gamma}$ simple. Let $P$ be a point in $S$. Then $P$ is $\bar{\Gamma}$-invariant. The action of the group $\bar{\Gamma}$ on the tangent space to the threefold $V_{14}$ at the point $P$ gives its faithful three-dimensional representation, which does not exist (see [15]).

Lemma A.13. The inequality $g \geqslant 11$ holds.

Proof. Suppose that $g \leqslant 10$. Then $g \leqslant 1$ by Theorem 2.18 since $|\bar{\Gamma}|=660$.

Moreover, it follows from the classification of finite subgroups of $\mathrm{PSL}_{2}(\mathbb{C})$ that the monomorphism $\theta$ does not exist if $g=0$. Thus, we see that $g=1$ and $\operatorname{Aut}(S)$ contains a simple non-abelian subgroup $\theta(\bar{\Gamma}) \cong \mathrm{PSL}_{2}\left(\mathbb{F}_{11}\right)$, which is impossible, because $\operatorname{Aut}(S)$ is solvable.

Using (A.9) and the Riemann-Roch theorem, we see that

$$
10 \geqslant h^{0}\left(\mathcal{O}_{\mathcal{L}\left(V_{14}, D\right)} \otimes \mathcal{O}_{V_{14}}(H)\right)=S \cdot H-g+1,
$$

because $\mathcal{L}\left(V_{14}, D\right)=Z$ and $2 g-2<S \cdot H$. Then $g \geqslant S \cdot H-9$. But $2 g-2<S \cdot H$. Hence $2(S \cdot H)-20 \leqslant 2 g-2<S \cdot H$, which implies that $S \cdot H \leqslant 19$. Therefore, $g \leqslant 10$, which is impossible by Lemma A.13.

The obtained contradiction shows that $V_{14}$ is $\bar{\Gamma}$-birationally superrigid.

To complete the proof of Theorem A.5, we assume that the threefold $V_{3}$ is not $\bar{\Gamma}$-birationally superrigid. Then there is a $\bar{\Gamma}$-invariant linear system $\mathcal{M}$ without fixed components such that $\lambda \mathcal{M} \sim \mathbb{Q}-K_{V_{3}}$ for some $\lambda \in \mathbb{Q}>0$. Then $\left(V_{3}, \lambda \mathcal{M}\right)$ is not canonical (see [7, Theorem 1.4.1]).

There is $\mu \in \mathbb{Q}$ such that $\mu<2 \lambda$ and $\left(V_{3}, \mu \mathcal{M}\right)$ is strictly log canonical.

Let $S \subset V_{3}$ be a minimal center in $\mathbb{L} \mathbb{C}\left(V_{3}, \mu \mathcal{M}\right)$. Then $\operatorname{dim}(S) \in\{0,1\}$.

By Lemma 2.13, there is a $\bar{\Gamma}$-invariant effective $\mathbb{Q}$-divisor $D$ on the threefold $V_{3}$ such that the set $\mathbb{L} \mathbb{C S}\left(V_{3}, D\right)$ consists of irreducible components of the $\bar{\Gamma}$-orbit of $S$, and $D \sim_{\mathbb{Q}}-\epsilon K_{V_{3}}$, where $\epsilon$ is a positive rational number such that $\epsilon<2$.

Let $\mathcal{I}$ be the multiplier ideal sheaf of the $\log$ pair $\left(V_{3}, D\right)$, and let $\mathcal{L}\left(V_{3}, D\right)$ be the log canonical singularities subscheme of the log pair $\left(V_{3}, D\right)$. Then it follows from Theorem 2.4 that

$$
\text { (A.14) } h^{0}\left(\mathcal{O}_{\mathcal{L}\left(V_{3}, D\right)} \otimes \mathcal{O}_{V_{3}}(2 H)\right)=h^{0}\left(\mathcal{O}_{V_{3}}(2 H)\right)-h^{0}\left(\mathcal{O}_{V_{3}}(2 H) \otimes \mathcal{I}\right)=15-h^{0}\left(\mathcal{O}_{V_{3}}(2 H) \otimes \mathcal{I}\right) \text {, }
$$

where $H$ is an ample generator of the group $\operatorname{Pic}\left(V_{3}\right)$.

Lemma A.15. The equality $\operatorname{dim}(S)=0$ is impossible. 
Proof. Suppose that $\operatorname{dim}(S)=0$. Let $\bar{F} \subset \bar{\Gamma}$ be a stabilizer of a point in $\operatorname{LCS}\left(V_{3}, D\right)$. Then

$$
|\bar{F}|=\frac{|\bar{\Gamma}|}{\left|\operatorname{LCS}\left(V_{3}, D\right)\right|} \geqslant \frac{|\bar{\Gamma}|}{15}=44
$$

by (A.14). Thus, if $\bar{F} \neq \bar{\Gamma}$, then $\bar{F}$ is isomorphic to either $\mathrm{A}_{5}$ or $\mathbb{Z}_{11} \rtimes \mathbb{Z}_{5}$ (see [15]).

We may identify $\bar{\Gamma}$ with a subgroup in $\operatorname{Aut}\left(\mathbb{P}^{4}\right)$. There is a subgroup $G \subset \mathrm{SL}_{5}(\mathbb{C})$ such that

$$
\bar{\Gamma}=\phi(G) \subset \operatorname{Aut}\left(\mathbb{P}^{4}\right) \cong \mathrm{PGL}_{5}(\mathbb{C})
$$

and $G \cong \bar{\Gamma}$, where $\phi: \mathrm{SL}_{5}(\mathbb{C}) \rightarrow \operatorname{Aut}\left(\mathbb{P}^{4}\right)$ is the natural projection.

Put $W=\mathbb{C}^{5}$. Then $W$ is an irreducible representation of the group $G$ (see [1]), which implies that $\bar{F} \neq \bar{\Gamma}$. If $F$ is a subgroup of the group $G$ such that $\phi(F)=\bar{F}$, then one can show that $W$ is an irreducible representation of the group $F$, which is a contradiction.

It follows from Theorem 2.8 that $S$ is a smooth curve of genus $g$ such that $g \leqslant S \cdot H$.

Lemma A.16. The curve $S$ is $\bar{\Gamma}$-invariant.

Proof. Let $Z$ be the $\bar{\Gamma}$-orbit of the curve $S$. Then $Z=\mathcal{L}\left(V_{3}, D\right)$, because $\left(V_{3}, D\right)$ is log canonical.

Suppose that $S \neq Z$. Let $r$ be the number of irreducible components of $Z$. Then $r \geqslant 11$, because there is no non-trivial homomorphism $\bar{\Gamma} \rightarrow \mathrm{S}_{r}$ in the case when $1<r \leqslant 10$.

Using (A.14) and the Riemann-Roch theorem, we see that

$$
15 \geqslant 15-h^{0}\left(\mathcal{O}_{V_{3}}(2 H) \otimes \mathcal{I}\right)=h^{0}\left(\mathcal{O}_{\mathcal{L}\left(V_{3}, D\right)} \otimes \mathcal{O}_{V_{3}}(2 H)\right)=r(2 S \cdot H-g+1),
$$

because $\mathcal{L}\left(V_{3}, D\right)=Z$ and $g \leqslant S \cdot H$. Since $r \geqslant 11$, one has $2 S \cdot H-g+1 \leqslant 1$, which contradicts the inequality $g \leqslant S \cdot H$.

We see that there is a natural homomorphism $\theta: \bar{\Gamma} \rightarrow \operatorname{Aut}(S)$. Arguing as in Lemma A.12, we see that $\theta$ is a monomorphism. Arguing as in Lemma A.13, one obtains $g \geqslant 11$.

By Theorem 2.18 we may assume that $g=14$. Let $a_{i}$ be the number of points on $S$ whose stabilizers in $\bar{\Gamma}$ are isomorphic to $\mathbb{Z}_{i}$. Using the Riemann-Hurwitz formula, we see that

$$
2 g-2=(2 \bar{g}-2) \cdot|\bar{\Gamma}|+330 a_{2}+440 a_{3}+528 a_{5}+550 a_{6}+600 a_{11},
$$

where $\bar{g}$ is the genus of the quotient curve $S / \bar{\Gamma}$ (cf. the proof of Lemma 2.10). Then $\bar{g}=0$. We have

$$
1294-528 a_{5}=330 a_{2}+440 a_{3}+550 a_{6}+600 a_{11},
$$

which leads to a contradiction. The obtained contradiction completes the proof of Theorem A.5.

\section{Appendix B. Del Pezzo fibrations}

\section{by Yuri Prokhorov}

Let $X$ be a threefold with at worst terminal singularities such that the group $\operatorname{Aut}(X)$ has a subgroup $\bar{G} \cong \mathrm{A}_{6}$, and let $\pi: X \rightarrow \mathbb{P}^{1}$ be a $\bar{G}$-Mori fibration.

The goal of this appendix is to prove the following result.

Theorem B.1. The isomorphism $X \cong \mathbb{P}^{1} \times \mathbb{P}^{2}$ holds, and $\pi$ is the projection to the first factor.

Recall that there exists no monomorphism $\bar{G} \rightarrow \mathrm{PGL}_{2}(\mathbb{C})$.

Lemma B.2 (cf. [38, Lemma 4.5]). Let $Y$ be a threefold with at worst terminal singularities such that $\operatorname{Aut}(Y)$ has a subgroup $\bar{G} \cong \mathrm{A}_{6}$. Then $Y$ contains no $\bar{G}$-invariant points.

${ }^{*}$ This work is partially supported by the grants RFBR 08-01-00395-a, NSh-1983.2008.1 and NSh-1987.2008.1. 
Proof. Suppose that $Y$ contains a $\bar{G}$-invariant point $P \in Y$. Let us show that this assumption leads to a contradiction. Let $T_{P, Y}$ be the Zariski tangent space to $Y$ at the point $P$. Then $\operatorname{dim}\left(T_{P, Y}\right) \geqslant 5$, because $\bar{G}$ has no faithful representations of dimension less than 5 . We see that $Y$ is not Gorenstein at the point $P$ (see [39, Section 3]). Let us regard $(Y \ni P)$ as an analytic germ.

Let $r$ be the Gorenstein index of the singularity $(Y, P)$, let $\pi:\left(Y^{\sharp}, P^{\sharp}\right) \rightarrow(Y, P)$ be the index one cover (see [39, Section 3.5]), where $P^{\sharp}=\pi^{-1}(P)$. Then

$$
\pi: Y^{\sharp} \backslash\left\{P^{\sharp}\right\} \rightarrow Y \backslash\{P\}
$$

is the topological universal cover (of degree $r$ ). Thus, there is an exact sequence of groups

$$
1 \longrightarrow \mathbb{Z}_{r} \longrightarrow \bar{G}^{\sharp} \stackrel{\beta}{\longrightarrow} \bar{G} \longrightarrow
$$

where $\bar{G}^{\sharp}$ is a finite subgroup in $\operatorname{Aut}\left(Y^{\sharp}, P^{\sharp}\right)$. Since $\bar{G}$ is simple, this is a central extension.

The group $\bar{G}^{\sharp}$ naturally acts on the Zariski tangent space $T_{P^{\sharp}, Y^{\sharp}}$ to $Y^{\sharp}$ at the point $P^{\sharp}$.

Recall that $\left(Y^{\sharp}, P^{\sharp}\right)$ is a hypersurface singularity. Hence, we have $\operatorname{dim}\left(T_{P^{\sharp}, Y^{\sharp}}\right) \leqslant 4$.

By the classification of three-dimensional terminal singularities (see [39, Section 6.1]) the action of the group $\alpha\left(\mathbb{Z}_{r}\right)$ on $T_{P^{\sharp}, Y^{\sharp}}$ in some coordinate system has one of the following forms:

- either $\operatorname{dim}\left(T_{P^{\sharp}, Y^{\sharp}}\right)=3$ and $\left(x_{1}, x_{2}, x_{3}\right) \longmapsto\left(\varepsilon x_{1}, \varepsilon^{-1} x_{2}, \varepsilon^{a} x_{3}\right)$,

- or $\operatorname{dim}\left(T_{P^{\sharp}, Y^{\sharp}}\right)=4$ and $\left(x_{1}, x_{2}, x_{3}, x_{4}\right) \longmapsto\left(\varepsilon x_{1}, \varepsilon^{-1} x_{2}, \varepsilon^{a} x_{3}, x_{4}\right)$,

- or $\operatorname{dim}\left(T_{P^{\sharp}, Y^{\sharp}}\right)=r=4$ and $\left(x_{1}, x_{2}, x_{3}, x_{4}\right) \longmapsto\left(\sqrt{-1} x_{1},-\sqrt{-1} x_{2}, \pm \sqrt{-1} x_{3},-x_{4}\right)$,

where $\varepsilon$ is a primitive $r$-th root of unity and $\operatorname{gcd}(r, a)=1$.

The case $\operatorname{dim}\left(T_{P^{\sharp}, Y^{\sharp}}\right)=3$ and $r=2$ is impossible, because the group 2.A 6 does not have faithful three-dimensional representations. Therefore, the central subgroup $\alpha\left(\mathbb{Z}_{r}\right) \subset \bar{G}^{\sharp}$ has at least 2 different eigenvalues. Then $T_{P^{\sharp}, Y^{\sharp}}$ is a reducible representation of the group $\bar{G}^{\sharp}$.

If $\operatorname{dim}\left(T_{P^{\sharp}, Y^{\sharp}}\right)=4$, then the subgroup $\alpha\left(\mathbb{Z}_{r}\right)$ has at least 3 different eigenvalues.

Hence, in every possible case, the group $\bar{G}^{\sharp}$ has a subrepresentation of dimension at least 2, which is impossible, because the group $\bar{G} \cong \mathrm{A}_{6}$ does no admit any embedding to $\mathrm{PGL}_{2}(\mathbb{C})$.

Lemma B.3 (cf. [16]). Let $S$ be a smooth del Pezzo surface such that $S$ admits a non-trivial action of the group $\bar{G}$. Then $S \cong \mathbb{P}^{2}$.

Proof. Note that $S \nsubseteq \mathbb{P}^{1} \times \mathbb{P}^{1}$, because there exists no monomorphism $\bar{G} \rightarrow \mathrm{PGL}_{2}(\mathbb{C})$.

Suppose that $S \neq \mathbb{P}^{2}$. Let us derive a contradiction.

If $K_{S}^{2} \geqslant 5$, then the action of $\bar{G}$ on $\operatorname{Pic}(S)$ is trivial, because $\operatorname{rk} \operatorname{Pic}(S) \leqslant 5$ and the canonical class $K_{S}$ is $\bar{G}$-invariant. Then any $(-1)$-curve on $S$ must be invariant, a contradiction.

Let $C$ be a $\bar{G}$-invariant curve in $\left|-K_{S}\right|$. Then every component of the curve $C$ is either rational or elliptic curve. Moreover, the curve $C$ consists of at most 4 components, which immediately implies that $C$ is not $\bar{G}$-invariant, because $\bar{G}$ is simple.

Put $V=H^{0}\left(\mathcal{O}_{S}\left(-K_{S}\right)\right)$. By the above, the group $\bar{G}$ acts non-trivially on $V$. Then

$$
4 \geqslant K_{S}^{2}=h^{0}\left(\mathcal{O}_{S}\left(-K_{S}\right)\right)-1 \geqslant 4,
$$

which implies that $K_{S}^{2}=4$, and the space $V$ is an irreducible five-dimensional representation of the group $\bar{G}$, because $\bar{G}$ has no non-trivial representations of dimension less than 5 (see [15]).

We see that $S=Q_{1} \cap Q_{2} \subset \mathbb{P}^{4}$, where $Q_{1}$ and $Q_{2}$ are irreducible quadric hypersurfaces.

The action of the group $\bar{G}$ on the space $V$ induces its action on $\mathbb{P}^{4}$.

Let $\mathcal{P}$ be the pencil generated by $Q_{1}$ and $Q_{2}$ is $\bar{G}$-invariant. Then $\mathcal{P}$ is $\bar{G}$-invariant. Since there is no monomorphism $\bar{G} \rightarrow \mathrm{PGL}_{2}(\mathbb{C})$, both quadrics $Q_{1}$ and $Q_{2}$ are $\bar{G}$-invariant, which is impossible, because otherwise the vertex of a degenerate quadric in pencil is a fixed point.

Corollary B.4. Let $F_{\pi}$ be a general fiber of the morphism $\pi$. Then $K_{F_{\pi}}^{2}=9$ 
Let $F$ be any scheme fiber of the morphism $\pi: X \rightarrow \mathbb{P}^{1}$. Then $F$ is $\bar{G}$-invariant.

Lemma B.5. The threefold $X$ is smooth and $F \cong \mathbb{P}^{2}$.

Proof. First we show that $\operatorname{Supp}(F)$ is irreducible, normal and has at worst Kawamata $\log$ terminal singularities. This step is similar to the proof of [34, Proposition 2.6].

Take $\mu \in \mathbb{Q}$ such that $(X, \mu F)$ is strictly log-canonical. Then there are a $\bar{G}$-invariant $\pi$-ample divisor $H$ and small positive $\delta_{1}$ and $\delta_{2} \in \mathbb{Q}$ such that $\left(X,\left(\mu-\delta_{1}\right) F+\delta_{2} H\right)$ is log canonical and

$$
\mathbb{L} \mathbb{C S}\left(X,\left(\mu-\delta_{1}\right) F+\delta_{2} H\right)=\bigcup_{g \in \bar{G}} g(S)
$$

where $S$ is a minimal center in $\mathbb{L} \mathbb{C S}(X, \mu F)$ (cf. Lemma2.13] or the proof of [34, Proposition 2.6]).

Put $D=\left(\mu-\delta_{1}\right) F+\delta_{2} H$. Then $\operatorname{LCS}(X, D)$ is a $\bar{G}$-orbit of the center $S$.

By Theorem [2.5, the locus $\operatorname{LCS}(X, D)$ is connected. It follows from Lemma 2.7 that $\mathbb{L} \mathbb{C} S(X, D)=S$, which implies that $S$ is not a point by Lemma B.2.

If $S$ is a curve, then $S \cong \mathbb{P}^{1}$ by Theorem 2.8, which is again impossible by Lemma B.2.

We see that $S$ is a $\bar{G}$-invariant surface. Then $S=\operatorname{Supp}(F)$, because $\pi$ is a $\bar{G}$-Mori fibred space.

By Theorem 2.8, the surface $S$ is normal and has Kawamata log terminal singularities.

Let $\Sigma \subset S$ be a subset consisting of points where $S$ is not Cartier. If $F$ is not reduced, then $1 \leqslant|\Sigma| \leqslant 4$ by [34, Theorem 1.1]. Since $\Sigma$ is $\bar{G}$-invariant, we see that $\Sigma=\varnothing$ by Lemma B.2.

We see that $F$ is a reduced normal surface having only quotient singularities, which implies that $F$ is a degeneration of $\mathbb{P}^{2}$ by Corollary B.4. Hence, the inequality $|\operatorname{Sing}(F)| \leqslant 3$ holds (see [32, Main Theorem], [20, Corollary 1.2]). So, the surface $F$ is smooth by Lemma B.2, which immediately implies that $F \cong \mathbb{P}^{2}$.

Proof of Theorem B.1. It follows from Lemma B.5 that every scheme fiber of the morphism $\pi$ is isomorphic to $\mathbb{P}^{2}$. By [3, Proposition V.4.1], there are integers $b \geqslant a \geqslant 0$ such that

$$
X \cong \operatorname{Proj}\left(\mathcal{O}_{\mathbb{P}^{1}} \oplus \mathcal{O}_{\mathbb{P}^{1}}(a) \oplus \mathcal{O}_{\mathbb{P}^{1}}(b)\right) .
$$

Recall that the action of the group $\bar{G}$ on the base $\mathbb{P}^{1}$ is trivial.

By Lemma B.2, every fiber of the morphism $\pi$ contains no $\bar{G}$-fixed points nor $\bar{G}$-invariant lines, which implies that $a=b=0$ and so $X \cong \mathbb{P}^{1} \times \mathbb{P}^{2}$.

\section{REFERENCES}

[1] A. Adler, On the automorphism group of a certain cubic threefold American Journal of Mathematics 100 (1978), 1275-1280

[2] E. Arbarello, M. Cornalba, P. Griffiths, J. Harris, Geometry of algebraic curves. Volume I Grundlehren der mathematischen Wissenschaften 267 (1985) Springer-Verlag, New York-Heidelberg

[3] W. Barth, C. Peters, A. Van de Ven, Compact complex surfaces Springer-Verlag, Berlin, 1984

[4] A. Beauville, Non-rationality of the symmetric sextic Fano threefold arXiv:math/1102.1255 (2011).

[5] W. Bosma, J. Cannon, C. Playoust, The Magma algebra system. I. The user language Journal of Symbolic Computation, 24 (1997), 235-265

[6] T. Breuer, Characters and automorphism groups of compact Riemann surfaces London Mathematical Society Lecture Note Series 280, Cambridge University Press, 2000

[7] I. Cheltsov, Birationally rigid Fano varieties Russian Mathematical Surveys 60 (2005), 875-965

[8] I. Cheltsov, Log canonical thresholds of del Pezzo surfaces Geometric and Functional Analysis, 18 (2008), 1118-1144

[9] I. Cheltsov, C. Shramov, Log canonical thresholds of smooth Fano threefolds Russian Mathematical Surveys 63 (2008), 73-180 
[10] I. Cheltsov, C. Shramov, On exceptional quotient singularities Geometry and Topology, 15 (2011), 1843-1882

[11] C. Clemens, P. Griffiths, The intermediate Jacobian of the cubic threefold Annals of Mathematics 95 (1972), 73-100

[12] A. Corti, Factorizing birational maps of threefolds after Sarkisov Journal of Algebraic Geometry 4 (1995), 223-254

[13] A. Corti, Singularities of linear systems and 3-fold birational geometry L.M.S. Lecture Note Series 281 (2000), 259-312

[14] A. Corti, K. E. Smith, J. Kollár, Rational and nearly rational varieties Cambridge Studies in Advanced Mathematics 92 (2004), Cambridge University Press

[15] J. Conway, R. Curtis, S. Norton, R. Parker, R. Wilson, Atlas of finite groups Clarendon Press, Oxford, 1985

[16] I. Dolgachev, V. Iskovskikh, Finite subgroups of the plane Cremona group Progress in Mathematics, Birkhauser, Boston 269 (2009), 443-548

[17] W. Feit, The current situation in the theory of finite simple groups Actes du Congrès International des Mathématiciens, Gauthier-Villars, Paris (1971), 55-93

[18] H. Finkelnberg, Small resolutions of the Segre cubic Indagationes Mathematicae 90 (1987), 261-277

[19] H. Finkelnberg, J. Werner, Small resolutions of nodal cubic threefolds Indagationes Mathematicae 92 (1989), 185-198

[20] P. Hacking, Yu. Prokhorov, Smoothable del Pezzo surfaces with quotient singularities Compositio Mathematica 146 (2010), 169-192

[21] C. Hacon, J. McKernan, Ch. Xu, On the birational automorphisms of varieties of general type arXiv:math/1011.1464 (2010)

[22] R. Hartshorne, Algebraic geometry Graduate Texts in Mathematics 52 (1977) Springer-Verlag, New York-Heidelberg

[23] B. Hunt, The geometry of some special arithmetic quotients Lecture Notes in Mathematics 1637 (Springer-Verlag, New York, 1996)

[24] V. Iskovskikh, Yu. Manin, Three-dimensional quartics and counterexamples to the Lüroth problem Matematiskii Sbornik 86 (1971), 140-166

[25] V. Iskovskikh, Yu. Prokhorov, Fano varieties Encyclopaedia of Mathematical Sciences 47 (1999) Springer, Berlin

[26] V. Iskovskikh, A. Pukhlikov, Birational automorphisms of multidimensional algebraic manifolds Journal of Mathematical Sciences 82 (1996), 3528-3613

[27] Y. Kawamata, On Fujita's freeness conjecture for 3-folds and 4-folds Mathematische Annalen 308 (1997), 491-505

[28] Y. Kawamata, Subadjunction of log canonical divisors II American Journal of Mathematics 120 (1998), 893-899

[29] J. Kollár, Singularities of pairs Proceedings of Symposia in Pure Mathematics 62 (1997), 221-287

[30] R. Lazarsfeld, Positivity in algebraic geometry II Springer-Verlag, Berlin, 2004

[31] N. Lemire, V. Popov, Z. Rechstein, Cayley groups Journal of the American Mathematical Society 19 (2006), 921-967

[32] M. Manetti, Normal degenerations of the complex projective plane Journal für die Reine und Angewandte Mathematik 419 (1991), 89-11

[33] M. Mella, Birational geometry of quartic 3-folds II: the importance of being $\mathbb{Q}$-factorial Mathematische Annalen 330 (2004), 107-126

[34] S. Mori, Yu. Prokhorov, Multiple fibers of del Pezzo fibrations Proceedings of the Steklov Institute of Mathematics 264 (2009), 131-145

[35] S. Mukai, Curves and symmetric spaces Proceedings of the Japan Academy, Series A, Mathematical Sciences 68 (1992), 7-10

[36] I. Nieto, The normalizer of the level $(2,2)$-Heisenberg group Manuscripta Mathematica 76 (1992), 257-267 
[37] K. Pettersen, On nodal determinantal quartic hypersurfaces in $\mathbb{P}^{4}$ Thesis, University of Oslo (1998)

[38] Yu. Prokhorov, Simple finite subgroups of the Cremona group of rank 3 Journal of Algebraic Geometry, to appear

[39] M. Reid, Young person's guide to canonical singularities Proceedings of Symposia in Pure Mathematics 46 (1987), 345-414

[40] J.-P. Serre, A Minkowski-style bound for the order of the finite subgroups of the Cremona group of rank 2 over an arbitrary field Moscow Mathematical Journal 9 (2009), 183-198

[41] V.Shokurov, Three-fold log flips Russian Academy of Sciences, Izvestiya Mathematics 40 (1993), 95-202

[42] C. Shramov, Birational automorphisms of nodal quartic threefolds arXiv:math/0803.4348 (2008)

[43] G. Tian, On Kähler-Einstein metrics on certain Kähler manifolds with $c_{1}(M)>0$ Inventiones Mathematicae 89 (1987), 225-246

[44] G. Tian, S.-T. Yau, Kähler-Einstein metrics metrics on complex surfaces with $\mathrm{C}_{1}>0$ Communications in Mathematical Physics 112 (1987), 175-203

[45] J. A. Todd, Configurations defined by six lines in space of three dimensions Mathematical Proceedings of the Cambridge Philosophical Society 29 (1933), 52-68

[46] D. Vazzana, Invariants and projections of six lines in projective space

Transactions of the American Mathematical Society 353 (2001), 2673-2688

University of Edinburgh, Edinburgh EH9 3JZ, UK

I.CHELTSOV@ED.AC.UK SHRAMOV@MCCME.RU 